San Jose State University

SJSU ScholarWorks

Master's Theses

Master's Theses and Graduate Research

Summer 2013

\title{
Experiences of coping with injury in Division I athletes from low- to-middle socioeconomic status backgrounds
}

Matthew Philip Bejar

San Jose State University

Follow this and additional works at: https://scholarworks.sjsu.edu/etd_theses

\section{Recommended Citation}

Bejar, Matthew Philip, "Experiences of coping with injury in Division I athletes from low-to-middle socioeconomic status backgrounds" (2013). Master's Theses. 4328.

DOI: https://doi.org/10.31979/etd.4r8m-nfez

https://scholarworks.sjsu.edu/etd_theses/4328

This Thesis is brought to you for free and open access by the Master's Theses and Graduate Research at SJSU ScholarWorks. It has been accepted for inclusion in Master's Theses by an authorized administrator of SJSU ScholarWorks. For more information, please contact scholarworks@sjsu.edu. 
EXPERIENCES OF COPING WITH INJURY IN DIVISION I ATHLETES FROM LOW-TO-MIDDLE SOCIOECONOMIC STATUS BACKGROUNDS

\author{
A Thesis \\ Presented to \\ The Faculty of the Department of Kinesiology \\ San José State University \\ In Partial Fulfillment \\ of the Requirements for the Degree \\ Master of Arts \\ by \\ Matthew P. Bejar
}

August 2013 
(C) 2013

Matthew P. Bejar

ALL RIGHTS RESERVED 
The Designated Thesis Committee Approves the Thesis Titled

EXPERIENCES OF COPING WITH INJURY IN DIVISION I ATHLETES FROM LOW-TO-MIDDLE SOCIOECONOMIC STATUS BACKGROUNDS

\author{
By \\ Matthew P. Bejar \\ APPROVED FOR THE DEPARTMENT OF KINESIOLGOY \\ SAN JOSÉ STATE UNIVERSITY
}

August 2013
Dr. Ted Butryn
Department of Kinesiology
Dr. Jessica Chin
Department of Kinesiology
Dr. Tamar Semerjian Department of Kinesiology 


\section{ABSTRACT \\ EXPERIENCES OF COPING WITH INJURY IN DIVISION I ATHLETES FROM LOW-TO-MIDDLE SOCIOECONOMIC STATUS BACKGROUNDS

\author{
by Matthew P. Bejar
}

Injuries inevitably occur in any sport at any level. The integrated sport injury model is one of the most extensive frameworks to address the psychological responses to athletic injury. While this model posits that socioeconomic status (SES) influences how an athlete cognitively, emotionally, and behaviorally responds to an injury, no research has substantiated this claim. Low SES individuals experience an exceptional amount of stress, which may complicate how they cope with a negative event. The coping strategies employed by an athlete can have major implications on rehabilitation adherence, recovery time, and psychosocial well-being. The purpose of this study was to qualitatively examine the experiences of low-to-middle SES athletes coping with injury. Semistructured interviews were conducted with 11 National Collegiate Athletic Association Division I athletes who had sustained recent injuries that lasted at least four weeks. A thematic data analysis produced 73 raw data themes and 16 higher-order themes, which were organized into five general dimensions: cognitive appraisals, emotional reactions, problem-focused coping, emotion-focused coping, and avoidance coping. While many participants initially reacted with catastrophizing thoughts and negatively toned emotions, they employed mostly adaptive forms of coping, such as positive reframing and perseverance. Generally, coaches, athletic trainers, sport psychology professionals, and other individuals should be more cognizant of SES and other related identities when collaborating with injured athletes. 


\section{ACKNOWLEDGMENTS}

There are many individuals to whom I would like to bestow my gratitude for helping make this project possible. I would like to thank Dr. Butryn, my thesis chair and advisor, for generously mentoring me and believing that I could aspire to a greater level than I had initially believed possible. I would also like to thank my thesis committee: Dr. Jessica Chin, for demanding nothing less than exemplary work, as well as introducing me to different ways of understanding and conducting research; and Dr. Tamar Semerjian, for also setting rigorous yet attainable standards and for introducing me to cultural sport psychology, an area where I hope to make contributions. Thank you to all of the athletic trainers who helped identify and recruit participants. Also, I am very thankful to the participants themselves who were willing to take the time to share their experiences, especially since some of these issues were of a sensitive and/or private matter. Last (but certainly not least), I would like to thank my parents for their love and patience over the years. Their unconditional support means a lot more than what I could hope to capture in words. 


\section{TABLE OF CONTENTS}

Chapter 1: INTRODUCTION 1

Statement of Purpose 5

Definitions 5

Delimitations $\quad 5$

Limitations 6

Chapter 2: LITERATURE REVIEW 8

Stress and Coping Frameworks 8

General adaptation syndrome $\quad 9$

Transactional model of stress and coping $\quad 9$

Two-dimensional coping conceptual framework $\quad 14$

Cognitive-motivational-relational theory of emotion $\quad 16$

Coping with Athletic Injury 18

The Influence of SES on Stress and Coping 26

Intersections among SES, Race, and Ethnicity 30

The Role of SES and Ethnicity in Sport 39

Chapter 3: METHODS $\quad 44$

Measures 44

Demographics $\quad 44$

SES $\quad 44$

Experiences of coping with injury $\quad 45$

Participants 46

Procedure 49

Data Analysis $\quad 51$

Academic Rigor $\quad 52$

Chapter 4: RESULTS $\quad 56$

Cognitive Appraisals $\quad 56$

Confusion $\quad 56$

Catastrophization $\quad 57$

Emotional Reactions $\quad 59$

Sadness $\quad 59$

Frustration $\quad 59$

Relief 61

Problem-focused Coping $\quad 61$

Comparison to past experiences 61

Commitment to rehabilitation 63

Perseverance $\quad 65$

Tangible support 66

Informational support 67 
Emotion-focused Coping $\quad 67$

Positive reframing 68

Use of religion $\quad 70$

Emotional support $\quad 71$

Avoidance Coping 72

Playing through pain $\quad 72$

Isolation $\quad 74$

$\begin{array}{ll}\text { Mental distraction } & 75\end{array}$

Chapter 5: DISCUSSION 76

Limitations $\quad 85$

Future Directions $\quad 87$

Conclusions and Implications 90

$\begin{array}{ll}\text { References } & 93\end{array}$

Appendix A: DEMOGRAPHIC INFORMATION 109

Appendix B: FAMILY AFFLUENCE SCALE II 110

Appendix C: INFORMED CONSENT FORM 111

Appendix D: INTERVIEW GUIDE 112

Appendix E: PARTICIPANT PROFILES 114

Appendix F: CONTENT ANALYSIS RESULTS 116

Appendix G: HUMAN SUBJECTS-INSTITUTIONALREVIEW 120

BOARD APPROVAL 


\section{Chapter 1}

\section{INTRODUCTION}

Injuries are unfortunate and inevitable occurrences in any sport. Athletes invest a great deal of time towards achieving optimal performance. Because their self-worth is often tied to these competition performances, they often perceive a long-term injury as a traumatic event (Quinn \& Fallon, 2000; Smith, Scott, \& Wiese, 1990). Intense training regimens, attractive incentives and rewards for successful athletes, dangerous terrain and climatic conditions, and cultures that glorify risk and minimize pain are examples of social contexts that enable injuries to occur (Pargman, 1999), especially in collegiate and professional sports. According to the National Collegiate Athletic Association (NCAA) Injury Surveillance data from 2004-2009, there were over 26,000 injuries in women's volleyball, 55,000 injuries in women's soccer, 55,000 injuries in men's soccer, 41,000 injuries in football, and 10,000 injuries in field hockey (National Collegiate Athletic Association, 2012a, 2012b, 2012c, 2012d, 2012e). Managing an athletic injury can be cognitively, behaviorally, and emotionally challenging (Pedersen, 1986). Injured athletes are subject to a number of psychological detriments, such as lowered self-esteem (Smith et al., 1990), mood disturbances (e.g., increased anger, depression, and anxiety; Carson \& Polman, 2008; O’Connor, 2011; Quinn \& Fallon, 1999, 2000; Udry, Gould, Bridges, \& Beck, 1997), helplessness (Carson \& Polman, 2008), social isolation (Henderson, 1999), fear of re-injury (Podlog \& Dionigi, 2010; Podlog \& Eklund, 2006), and many others. Consequently, understanding the psychological aspects of injury in addition to the physical factors is crucial, as athletes work to rehabilitate and return to play. 
Several authors in the field of sport psychology have examined both the psychological predictors of injury (Andersen \& Williams, 1988; Williams \& Andersen, 1998) and the post-injury responses of athletes (Bianco, Malo, \& Orlick, 1999; Mitchell, 2011; Hagger, Chatzisarantis, Griffin, \& Thatcher, 2005; Quinn \& Fallon, 1999, 2000; Rees, Evans, Mitchell, \& Hardy, 2010; Tracey, 2003; Wiese-Bjornstal, Smith, \& LaMott, 1995; Wiese-Bjornstal, Smith, Shaffer, \& Morrey, 1998). The integrated sport injury model (Wiese-Bjornstal et al., 1998) is one of the most comprehensive frameworks to address the psychological responses to athletic injury and rehabilitation. This model posits that several situational factors (e.g., type of sport, level of competition, scholarship status, access to rehabilitation services, family influences) and personal factors (e.g., type of injury, history of injuries, personality gender, age, ethnicity, socioeconomic status [SES]) influence the cognitive, emotional, and behavioral reactions of an athlete. They include the coping strategies that an athlete employs during rehabilitation. Over the past 15 years, numerous individuals (e.g., Albinson \& Petrie, 2003; Bianco et al., 1999; Quinn \& Fallon, 1999; Rees et al., 2010; Tracey, 2003) have investigated various components of the model; however, the present investigation focused on the athlete's responses to injury within the context of his or her socioeconomic background.

While Wiese-Bjornstal et al. (1998) list SES as one of several personal factors that influence an athlete's response to injury, no research has investigated this assertion. Generally, individuals from low SES backgrounds are likely to encounter more stress than their high SES counterparts (Chibnall, Tait, Andresen, \& Hadler, 2005; Kristenson, Eriksen, Sluiter, Starke, \& Ursin, 2004; Santiago \& Wadsworth, 2009; Silk \& Andrews, 
2012), which presents challenges when such individuals are confronted with a negative life event, such as a long-term athletic injury. The athlete's cognitive appraisal of the injury (i.e., perception of injury as a stressor) and subsequent coping strategies can have a profound impact on his or her psychosocial well-being and physical recovery (WieseBjornstal et al., 1995, 1998). Some of the more well-known frameworks that have represented stress and coping include Selye's (1974) model of stress adaptation, Lazarus and Folkman's (1984) transactional model of stress and coping, Anshel, Williams, and Hodge's (1999) two-dimensional framework of coping, and Lazarus' (2000) cognitivemotivational-relational model of emotion. Lazarus and Folkman (1984) posited that coping is dynamic and specific to a particular context. They proposed two general categories of coping strategies: problem-focused coping (i.e., addressing the situation that is causing the stress) and emotion-focused coping (i.e., addressing the emotions that arise from the perceived stressor). Anshel et al. (1999) contended that there were also two primary dispositional coping styles: approach (i.e., increasing one's understanding or control of the stressful situation) and avoidance (i.e., evading the perceived stressor). Other researchers (Endler \& Parker, 1990; Kowalski \& Crocker, 2001) have argued that avoidance coping is a third distinct coping strategy rather than a coping style. The influence of SES on these coping strategies is a potentially vital yet strikingly underdeveloped area of research in sport and exercise psychology. Coaches, athletic trainers, physiotherapists, sport psychology professionals, and other personnel need to be educated and well-equipped to engage the needs of diverse populations. 
Attempts to include the experiences of marginalized groups in the sport psychology literature have been, until quite recently, rare. Mann and Kato (1996) noted that certain populations pertaining to age, gender, ethnic group, SES, sexual orientation, and other types of groups have been marginalized within the health psychology literature in two main ways: 1) they have been excluded from samples, or 2) they have been included, but there has been no mention of ethnic differences. Ram, Starek, and Johnson (2004) echoed similar sentiments in regards to sport psychology research. These authors conducted a content analysis of 986 manuscripts from the Journal of Sport and Exercise Psychology, Journal of Applied Sport Psychology, and The Sport Psychologist and found that only $19.86 \%$ of articles referenced race or ethnicity and that $1.22 \%$ referenced sexual orientation. However, $77.05 \%$ of these articles only referenced race or ethnicity as characteristics of the samples used rather than addressing issues pertaining to race and ethnicity. Similarly, Kamphoff, Gill, Araki, and Hammond (2010) conducted a content analysis of 5,214 abstracts from the Association of Applied Sport Psychology (AASP) conference from 1986 to 2007 and found that only 10.5\% included a discussion of a cultural diversity issue, and only $31.9 \%$ of abstracts included a diverse sample. As Ryba and Wright (2005) advocated, sport psychologists not only have a duty to facilitate enhanced performance, but they should also be instrumental in promoting overall satisfaction and empowerment of the athlete. The sport psychology literature has tended to lag behind other disciplines, such as sport sociology or women's studies, in exploring these social justice issues; therefore, this study will help fill this large void in the research. 


\section{Statement of Purpose}

The purpose of this study was to examine the experiences of low-to-middle SES athletes coping with injury via qualitative interviews.

\section{Definitions}

1. Stress refers to "a particular relationship between the person and the environment that is appraised by the person as taxing or exceeding his or her resources and endangering his or her well-being" (Lazarus \& Folkman, 1984, p. 19).

2. Coping is defined as "constantly changing cognitive and behavioral efforts to manage specific external and/or internal demands that are appraised as taxing or exceeding the resources of the person" (Lazarus \& Folkman, 1984, p. 141).

3. Socioeconomic status (SES) refers to an individual or group's social location within the structure of society, which is determined by access to power, privilege, desirable resources, and rewards (Williams \& Rucker, 1996).

4. Race is a socially constructed concept entailing fluid classifications that signify social conflicts and interests and infer meanings based upon perceived physical differences (Omi \& Winant, 1994).

5. Ethnicity is defined as "a cultural heritage that people use to identify a particular population...based on cultural traditions and history" (Coakley, 2009, p. 276).

\section{Delimitations}

This study was delimited to the following instruments:

1. Questionnaire that assessed information on participant's demographics and injury (Appendix A). 
2. Family Affluence Scale II (FAS II; Currie et al., 2004; Appendix B).

3. Interview guide (Appendix D).

The study was delimited to the following participants:

1. NCAA Division I athletes.

2. Athletes residing in the San Francisco Bay Area.

3. Athletes who sustained an injury, which caused them to miss at least four weeks of full practice and/or competition, within a year of Institutional Review Board (IRB) approval.

4. Individuals from low-to-middle SES backgrounds, assessed by a score between "0" and "5" on the FAS II (Currie et al., 2004).

\section{Limitations}

The limitations of this study included the following:

1. The participants were at various stages in their recovery when the interviews took place. Due to the time-sensitive nature of this project, data were not collected at multiple points (i.e., different phases of the rehabilitation period) as other studies have done (e.g., Albinson \& Petrie, 2003; Carson \& Polman, 2008, 2010; Quinn $\&$ Fallon, 1999, 2000). These longitudinal designs have allowed one to observe how an athlete's coping strategies change throughout a period of time. Lazarus (1993) noted that coping is a dynamic process rather than a static variable, and this study may not have been able to clearly demonstrate this idea. 
2. The interviews were retrospective; hence, there may have been some prior experiences related to stress and coping that the participants had but could not recall during the interview.

3. The tangible support that NCAA Division I schools typically provide (e.g., paying medical expenses, providing personnel and equipment to facilitate rehabilitation) may have buffered some of the stress experienced by the participants.

4. There was a severe gender imbalance (nine males, two females); thus, the data may not have been representative of female athletes' experiences. 


\section{Chapter 2}

\section{LITERATURE REVIEW}

Injuries are likely to occur across all types and ability levels of sport. While the physical factors are certainly important, the psychological aspects are often ignored by coaches, athletic trainers, medical personnel, and other individuals working with injured athletes. Psychological factors are important in examining both the injury antecedents (Andersen \& Williams, 1988; Williams \& Andersen, 1998) and the post-injury responses of an athlete (Bianco et al., 1999; Mitchell, 2011; Hagger et al., 2005; Quinn \& Fallon, 1999, 2000; Rees et al., 2010; Tracey, 2003; Wiese-Bjornstal et al., 1995; WieseBjornstal et al., 1998). The present study primarily focused on post-injury responses, particularly coping strategies, of low-to-middle SES athletes. This review addresses: (a) stress and coping frameworks, (b) coping with athletic injury, (c) the influence of SES on stress and coping, (d) intersections among SES, race, and ethnicity, and (e) the role of SES and ethnicity in sport.

\section{Stress and Coping Frameworks}

Although there is near universal agreement on Lazarus and Folkman's (1985) definition of coping, there are various models of how stress and coping are represented in both the general and sport psychology literatures. The following are relevant for the present study and are highlighted below: (a) Selye's (1974) model of stress adaptation, (b) Lazarus and Folkman's (1984) transactional model of stress and coping, (c) Anshel and colleagues' (1997) two-dimensional framework of coping, and (d) Lazarus's (2000) cognitive-motivational-relational model of emotion. 
General adaptation syndrome. Selye (1983) defined stress from a physiological perspective as a "nonspecific response of the body to any demand made upon it" (p. 2). Selye's (1974) model of stress adaptation, which he called general adaptation syndrome, is one of the more recognized theories addressing the psychophysiological consequences of stress. He proposed that when individuals are exposed to a stressor, the initial response is alarm. If the intensity and duration of the stressor is overwhelming, then exhaustion will occur. If it is not overwhelming, an adaptation phase will occur where individuals become stronger than before. Applying this framework to the present study, the number and severity of stressors experienced by low SES individuals may overwhelm their coping resources; however, these athletes may also be more prepared to cope with the setbacks of a long-term injury. Accordingly, these stressors may facilitate positive, negative, or mixed influences on how they cope with an injury. Selye also identified two different types of stress: eustress and distress. Eustress refers to "good stress" which individuals must adapt to (e.g., promotion to the starting lineup on a sports team); whereas, distress refers to "bad stress" which individuals must adapt to (e.g., chronic injury).

Transactional model of stress and coping. Prior to Lazarus and Folkman's (1984) definition of coping, many ambiguous and flawed coping frameworks had been proposed. Lazarus and Folkman defined coping as "constantly changing cognitive and behavioral efforts to manage specific external and/or internal demands that are appraised as taxing or exceeding resources of the person" (p. 141). Over the past 25 years, this has 
been the conventional representation of coping in the sport psychology literature, as well as other fields.

One of the main contributions of this definition was the idea that coping is a process rather than a disposition (i.e., trait-oriented). Lazarus and Folkman (1984) proposed three main features that are associated with this process approach to coping. First, observations of coping are concerned with what the person actually thinks or does, rather than what he or she usually does, would do, or should do. Second, what the person actually thinks or does is examined within a specific context. In other words, coping strategies often vary across different situations that are perceived as stressful. Third, this process approach implies that changes in coping (i.e., both thoughts and actions) occur as the stressor unfolds. Individuals may rely on one type or category of coping in early stages, while they may utilize another strategy during the later stages. For example, an injured athlete may use more emotion-focused forms of coping during the initial period of injury, but depend on problem-solving strategies in the later phases of rehabilitation. Likewise, the functions of coping vary greatly according to the situation. In brief, coping is not a fixed state; instead, it represents a complex and dynamic process.

Although the particular phases of coping differ according to the context and perceived stressor, Lazarus and Folkman (1984) identified three general stages: anticipation, impact or confrontation, and postimpact. In the anticipation stage, the stressful event has not occurred yet. The cognitive appraisal process entails an evaluation of whether a stressful event will happen, when the stressful event will happen, and what will happen. Simultaneously, a secondary appraisal process occurs where the individual 
evaluates his or her sense of control. Here, the individual assesses whether, to what extent, and how he or she can manage the threat. During the impact stage, the thoughts and actions relevant to one's sense of control are no longer present since the stressful event has already occurred. The individual evaluates the perceived magnitude of the stressor to what he or she previously anticipated. When the person finds unexpected differences in control over the unfolding event, he or she must reassess its significance, a phenomenon known as reappraisal. In the postimpact stage, the same cognitive processes that started in the impact stage remain at work. Moreover, the stressful event encourages a host of new anticipatory processes. In other words, the individual examines how he or she will appraise stressors in the future. During the encounter with the stressor, the individual is learning about the realities of the unpleasant event and what can be done about it, which will have an impact on the person's coping strategies in the future. For example, if a football player learns he cannot control how hard someone tackles him, he may choose to focus on regulating his emotions in future circumstances. These three general stages are not mutually exclusive, and the boundaries between them are permeable.

Lazarus and Folkman (1984) identified two general coping strategies: problemfocused coping and emotion-focused coping. Problem-focused coping strategies address the conditions causing the stressful event and "are often directed at defining the problem, generating alternative solutions, weighing the alternatives in terms of their costs and benefits, choosing among them, and acting" (Lazarus \& Folkman, 1984, p. 152). These can be further classified into strategies directed inward, or at the self, and strategies 
directed at the environment (Kahn, Wolfe, Quinn, Snoek, \& Rosenthal, 1964). Strategies directed at the self, such as shifting the level of aspiration, finding alternative channels of gratification, reducing ego involvement, developing new standards of behavior, and learning new skills or procedures are aimed at making motivational and cognitive changes. Strategies directed at the environment seek to alter environmental pressures, barriers, resources, procedures, and other external factors. Other than developing new behavior and learning new skills, Lazarus and Folkman (1984) stated that the inwarddirected strategies represent cognitive reappraisals.

Emotion-focused strategies regulate emotions underlying the cognitively appraised stressful event. Typically, these are aimed at reducing emotional distress, and examples include avoidance, minimization, distancing, selective attention, positive comparisons, and wresting positive value from negative events (Lazarus \& Folkman, 1984). Nonetheless, there are some circumstances when individuals deliberately increase their emotional distress in order to mobilize themselves for action, such as increasing arousal before a competition. Some cognitive forms of emotion-focused coping, such as positive comparisons or seizing positive value from negative events, lead to reappraisal of a situation without changing the objective situation. Other strategies, such as substance use, meditating, exercise, and seeking emotional support, do not change the meaning of an event directly; although, it is possible these may indirectly lead to reappraisals of the situation. The meaning of the event may remain unchanged even if some aspects are focused on more closely or filtered out. Lazarus and Folkman (1984) affirmed that there is generally a wider array of emotion-focused coping strategies that 
can be applied universally across various situations compared to problem-focused coping strategies. Also, they noted that problem- and emotion-focused coping strategies have the potential to facilitate, impede, or have no effect on each other depending on the context. Likewise, one group of coping strategies is not always preferable over the other. Lazarus (1993) noted that emotion-focused coping strategies dominate when the situation is largely unchangeable; whereas, problem-focused coping strategies are more likely to occur when the situation is perceived as controllable.

Taking these key concepts together, Lazarus and Folkman (1984) developed a transactional model of stress and coping. Contrary to unidirectional models, this model suggested that the person and the environment are in a dynamic, mutually reciprocal, bidirectional relationship. When an individual encounters an environmental event, he or she makes an initial evaluation (primary appraisal) of the situation. If the person determines there is no threat, then no stress occurs. If the person perceives a threat, he or she evaluates the controllability of the situation, coping resources, and effectiveness of these options (secondary appraisal). The person who perceives he or she can cope with the threat experiences positive stress. The individual who perceives he or she cannot cope with the threat experiences negative stress. The use of problem-focused and/or emotion-focused coping strategies mediate the relationship between the secondary appraisal and the stress that is experienced such that certain coping behaviors alter the behavioral responses (e.g., alter the environment) and/or psychological responses (e.g., alter cognitions, defenses, etc.). In turn, the psychological responses impact one's physiological responses (e.g., heart rate, blood pressure, immune function, etc.). 
Together, the physiological, psychological, and behavioral responses may revise the primary appraisal of the stressor.

Two-dimensional coping conceptual framework. In addition to the coping strategies proposed by Lazarus and Folkman (1984), other researchers (Carver, Scheier, \& Weintraub, 1989; Compas, 1987; Endler \& Parker, 1990; Krohne, 1993; Roth \& Cohen, 1986) have called attention to coping styles. Coping styles are "methods of coping that characterize individuals' reactions to stress either across different situations or over time within a given situation" (Compas, 1987, p. 394). Coping strategies represent dynamic concepts that vary across situations; whereas, coping styles are dispositional attributes that can be predicted over multiple contexts. The two most prominent coping styles that have been discussed in the general psychology and sport psychology literature are approach coping and avoidance coping. Approach coping refers to "strategies characterized by intensified intake and processing of threatening information" (Krohne, 1993, p. 21). The main objective of this coping style is to enhance one's understanding or control over the situation in order to lessen the negative effects of a stressful event (Anshel, 2001). Examples include initiating direct action, increasing one's efforts to reduce stress intensity, and pre-planning a coping strategy. Roth and Cohen (1986) stated that approach coping is preferable when the individual views the situation as controllable, knows the source of stress, is receptive to discussing the situation, has adequate time to resolve or address the issue, and will be disadvantaged by failing to resolve the problem. 
Avoidance coping refers to physically or mentally "turning away from the threatrelated cues" (Krohne, 1993, p. 21). Possible reasons for an individual to use avoidance coping are to distract him or herself from the stressor or to replace unpleasant thoughts with more positive ones (Anshel, 2001). Examples include avoiding a stressor by seeking out other people, ignoring or discounting the importance of a stressor, or by engaging in another task. Roth and Cohen (1986) stated that avoidance coping is preferable when the situation is uncontrollable, emotional resources are limited, the source of stress is ambiguous, the situation requires fast decision-making, and outcome measures are immediate or short-term.

Anshel et al. (1999) reconciled these dispositional (i.e., coping styles) and dynamic (i.e., coping strategies) views of coping by proposing a two-dimensional coping conceptual framework with approach and avoidance dimensions, as well as problemfocused and emotion-focused sub-dimensions. Taken together, the four resulting categories are: (a) approach/problem-focused, (b) approach/emotion-focused, (c) avoidance/problem-focused, and (d) avoidance/emotion-focused. An example of an approach/problem-focused strategy is analyzing why mistakes were made and making efforts to correct them. An example of an approach/emotion-focused coping strategy may be using progressive relaxation to reduce stress. An avoidance/problem-focused strategy may be applying a mental distraction to avoid thinking about the problem. An example of an avoidance/emotion-focused coping strategy is venting unpleasant emotions. While this is a popular model in the stress and coping research, it has not achieved universal agreement. For instance, other researchers (Endler \& Parker, 1990; 
Kowalski \& Crocker, 2001) have argued that avoidance coping is a distinct coping strategy rather than a coping style. Anshel (2001), one of the developers of the aforementioned model, has even suggested that behavioral and cognitive coping strategy sub-dimensions (rather than problem-focused and emotion-focused) may be more useful for studying the coping responses to acute stressors in sport. Generally, there has not been an overwhelming consensus on a set of coping categories, which has been a challenge for this area of research (Amirkhan \& Auyeung, 2007; Skinner, Edge, Altman, \& Sherwood, 2003).

Cognitive-motivational-relational theory of emotion. Lazarus (2000) noted that competitive sport represents an ideal context to examine stress and coping. $\mathrm{He}$ argued that stress is often examined as a one-dimensional concept, which fails to account for the underlying emotions. More specifically, he called attention to the role of emotions in athletic performance. Lazarus's (2000) cognitive-motivational-relational theory is helpful in understanding this phenomenon. This theory encompasses three main principles. First, there are many inter-individual and intra-individual differences to how people emotionally respond to similar situations; however, each stressful encounter shares a relational theme. These are harm, threat, challenge, or benefit. These relational meanings are perceived as gains (i.e., challenge, benefit) or losses (i.e., threat, harm). Second, the type and intensity of emotions that are experienced depends on one's cognitive appraisal, or interpretation, of the event's significance, as well as coping. A cognitive appraisal consists of both cognitive components (e.g., beliefs about self and world, resources) and motivational components (e.g., individual goals, priority of goals). 
Emotions can facilitate adaptation if the perception is realistic, but emotions can also encourage maladaptive behaviors if the appraisal is unrealistic. In addition to the cognitive appraisals, coping can influence which emotions will arise and how they will change. For example, an individual may feel less angry and more relieved after venting his/her frustrations to a friend. In other words, the coping strategies that an individual employs throughout a stressful encounter will impact the emotions experienced during this process. Third, given the wide array of aspects involved in the cognitive appraisal process, including personal factors and environmental demands, constraints, and opportunities, the constructed relational meaning is unique to each emotion.

Relating this theory to sport, Lazarus (2000) asserted that there were three psychological processes essential to performance that could be influenced by emotions: (a) motivation, or the commitment of energy and persistence in both practice and competition; (b) focus on what is occurring in competition; and (c) concentration on the actions and strategies required to produce a favorable outcome. Emotions may facilitate or hinder these goals. For example, emotions can serve to increase arousal and motivation during competition; however, emotions can also distract an individual from performing the necessary tasks to be successful. The particular form of an emotion influences the way it impacts performance. For example, one particular form of anger could serve to increase an athlete's motivation to score a goal; whereas, another form of anger may enable an athlete to pout about a bad call and lose focus of necessary tasks. Additionally, emotions may arise from other contexts, which may play a role in sport. 
For example, emotions that arise from family circumstances may impact how an athlete performs during competition.

\section{Coping with Athletic Injury}

Athletes often perceive injuries, especially those of a long-term nature, as major stressors. When an individual is restricted from participating in his or her desired choice of physical activity, he or she is unable to fully enjoy the benefits of sport and/or exercise, such as improved self-esteem, increased competence, decreased depression, and others (Smith et al., 1990). In particular, elite athletes engage in ample physical and mental preparation in order to attain optimal performance (Quinn \& Fallon, 1999); thus, an athlete may struggle to cope when he or she is no longer able to train or compete. As a result, injured athletes are subject to a number of psychological detriments, such as lowered self-esteem (Smith et al., 1990), mood disturbances (e.g., increased anger, depression, and anxiety; Carson \& Polman, 2008; O'Connor, 2011; Quinn \& Fallon, 1999, 2000; Udry et al., 1997), helplessness (Carson \& Polman, 2008), social isolation (Henderson, 1999), and many others. These setbacks are magnified in athletes whose injuries result in disability. These athletes frequently face marginalizing organizational policies, discrimination, and social attitudes (Rimmer, 2005), which often lead to diminished self-worth and lower optimism (Stephens, Neil, \& Smith, 2012). Given these setbacks, sport psychology professionals, athletic trainers, coaches, medical practitioners, and other personnel must be able to understand and address the cognitive, emotional, and behavioral challenges that athletes face during rehabilitation (Pedersen, 1986; Smith et al., 1990). 
The integrated sport injury model (Wiese-Bjornstal et al., 1998) has been one of the most extensive frameworks to address the psychological responses to athletic injury and rehabilitation. This model suggests that many situational factors and personal factors influence the initial cognitive appraisal of an injury, which then leads to particular emotional responses that an athlete exhibits. In turn, the cognitive appraisal and emotional responses impact the athlete's behavioral responses during rehabilitation. The cognitive appraisal, or initial interpretation, entails concepts such as goal adjustment, beliefs and attributions, self-perceptions, and appraisal of coping skills. Emotional responses may include tension, anger, depression, positive outlook, and emotional coping skills. Relevant behavioral responses include adherence to rehabilitation, acceptance or rejection of social support, risk-taking behaviors, malingering, and other behavioral coping strategies. Athletes' coping skills are intertwined within the cognitive, emotional, and behavioral components of this model.

The situational factors that influence these injury responses include the sport (e.g., type of sport, level of competition, time in season, scholarship status), social influences (e.g., teammate, coach, family, sports medicine personnel, personal philosophy), and environmental factors (e.g., rehabilitation environment, access to rehabilitation services). Personal factors that influence the injury responses include the injury (e.g., type of injury, history of injuries, perceived cause, severity of injury, recovery status), psychological differences (e.g., personality, self-perceptions, pain tolerance), physical differences (e.g., use of ergogenic aids, physical health status), and demographic differences (e.g., gender, age, ethnicity, SES). 
Accordingly, Wiese-Bjornstal et al. $(1995,1998)$ noted that the athlete's cognitive, behavioral, and emotional responses are associated with his or her physical and psychosocial recovery outcomes. Ample research has substantiated this point. For example, problem-focused coping has been associated with rehabilitation attendance and adherence (Hagger et al., 2005). Also, instrumental coping (i.e., attempts to ease stress by becoming educated about the injury or seeking the advice of health-care providers) has been associated with faster rehabilitation times (Quinn \& Fallon, 2000). Furthermore, injured athletes who utilize social support have been shown to cope more effectively with the demands of rehabilitation (Green \& Weinberg, 2001; Rees et al., 2010). Clearly, the coping strategies that athletes employ carry significant implications for the outcome of their injuries.

Many researchers have investigated the coping strategies that athletes employ during the injury process using both quantitative instruments (Albinson \& Petrie, 2003; Quinn \& Fallon, 1999, 2000) and qualitative interviews (Bianco et al., 1999; Carson \& Polman, 2010; Ford \& Gordon, 1999; Gould, Udry, Bridges, \& Beck, 1997; Podlog \& Eklund, 2006). Injured athletes have used problem-focused coping strategies, such as gathering information on the recovery and rehabilitation processes (Carson \& Polman, 2008), instrumental coping (Bianco et al., 1998; Hoar \& Flint, 2008; Udry, 1997), and active planning (Hagger et al., 2005; Quinn \& Fallon, 1999, 2000). Related to this category of coping strategies, goal setting has been found to be an effective coping strategy, particularly in the later phases of rehabilitation when the athlete is preparing to return to play (Carson \& Polman, 2008; Hagger et al., 2005). Injured athletes have also 
used a wide array of emotion-focused coping strategies, which include venting of emotions (Gould et al., 1997; Hagger et al., 2005), seeking emotional social support (e.g., encouragement from coaches, teammates, and medical personnel; Carson \& Polman, 2008; Gould et al., 1997; Hagger et al., 2005; Hoar \& Flint, 2008; Manuel et al., 2002; Quinn \& Fallon, 1999), using positive self-talk (Carson \& Polman, 2008; Gould et al., 1997; Quinn \& Fallon, 2000), and reframing negative thoughts and emotions to positive attitudes (i.e., positive reinterpretation or positive reframing; Bianco et al., 1998; Gould et al., 1997; Quinn \& Fallon, 1999, 2000).

Additionally, injured athletes have used avoidance coping strategies that include isolating themselves from others (Carson \& Polman, 2010; Gould et al., 1997), keeping busy with activities not related to their sport (Carson \& Polman, 2008, 2010; Gould et al., 1997), exhibiting denial and/or tolerating the pain (Carson \& Polman, 2008; 2010; Quinn \& Fallon, 1999; Tracey, 2003), spending time with family (Carson \& Polman, 2008, 2010), and thought stopping (e.g., avoiding injury as a subject of conversation; Carson \& Polman, 2010). Other forms of avoidance coping may be categorized under Anshel and colleagues' (1997) two-dimensional model. For example, Quinn and Fallon (1999) found that athletes may employ behavioral disengagement (i.e., giving up trying to deal with a problem), mental disengagement (i.e., mentally distracting oneself from thinking about the problem), or substance abuse to cope with an injury. Behavioral disengagement may be categorized as avoidance/problem-focused; while, mental disengagement may be classified as avoidance/emotion-focused under the assumption that the intent is to prevent negative feelings from arising. Palliative coping, a less common strategy used by injured 
athletes which entails using self-help exercises to reduce the stress of the situation (Udry, 1997), may also be classified as avoidance/emotion-focused. Certain forms of avoidance coping, such as denial, are more ambiguous with regard to being classified as avoidance/problem-focused or avoidance/emotion-focused, which supports the view that avoidance coping is its own distinct coping strategy.

Other examples of coping strategies utilized by injured athletes that have been mentioned in the literature include turning to religion (Gould, Eklund, \& Jackson, 1992; Quinn \& Fallon, 1999), using humor (O’Connor, 2011), and learning valuable lessons from the experience (Gould et al., 1997). Their precise categorizations may overlap among problem-focused, emotion-focused, and avoidance coping strategies.

While Wiese-Bjornstal et al. (1998) proposed that SES and ethnicity impact the psychological response to sport injury, there has been very minimal research to date examining this potential influence. An extensive literature search revealed that Nixon (1996) is one of the few individuals to explore parts of this topic. He examined how gender, race, and four sport status variables (individual or team sport, contact or noncontact sports participation, status as a lineup regular, and status as a scholarship regular) predicted sports-related pain and injury pain and attitudes in 156 NCAA Division I athletes who had experienced significant injuries. This was a sociological analysis; hence, coping was not a central focus of this study. Nonetheless, one interesting finding that was relevant to the present investigation was that race modestly explained post-injury behavior (measured by questionnaires developed by the author) such that White athletes were more likely to be complicit with pressures from coaches and fans to play through 
their injuries. Nixon (1996) acknowledged that more research was needed with regard to race and this particular behavior, which may be considered a form of behavioral and/or avoidance coping. Another rare exception is the work of Plaatjie and Potgieter (2011), who examined the role of environment, ethnicity, and culture in coping with stress before, during, and after competition in 33 South African professional football (soccer) players. Through semi-structured interviews, the authors found that non-White athletes were exposed to many barriers associated with a lower socioeconomic position, such as absence of family support structures, home circumstances, poverty, and substance abuse, which hindered coping in these participants. In contrast, White players identified intrapersonal variables, such as personality differences, high expectations, and communication problems that complicated their abilities to cope with acute stressors in sport. Although Plaatjie and Potgieter (2011) were among a very small group of researchers who have looked at diversity issues within the context of stress and coping in sport, there was no mention of athletic injuries in their study.

Additionally, Anshel has conducted several studies on the influences of gender and culture on how athletes cope with acute stressors during competition. Anshel et al. (1997) looked at differences in coping among American males, American females, Australian males, and Australian females using the Coping Style in Sport Survey, which measured coping styles in response to acute stressors experienced during competition. When experiencing a bad call, females, particularly Australian females, preferred a psyching up strategy (approach/emotion-focused coping) more than males. In response to opponents cheating, females were less likely to complain, but they felt more frustrated 
than males. Females were more likely to ignore a cheating component. Similar to how they managed a bad call, females were more likely to use approach/emotion-focused coping strategies for these circumstances. After experiencing intense pain or injury, males were more likely to demonstrate avoidance/problem-focused coping strategies (i.e., ignore pain and continue to play) than females, who were more likely to use approach/emotion-focused coping strategies (e.g., using psyching up strategies). Australians were less concerned with their opponent's performance (avoidance/emotionfocused coping), and Australian females, in particular, wanted to focus on their own performance (approach/problem-focused coping).

In another study, Anshel, Sutarso, and Jubenville (2009) examined the effect of gender (male and female) and race (Caucasian, African American, and Hispanic) on coping with acute stressors in sport using the Coping Style in Sport Scale. Caucasians reported the most coach-related and performance-related stress. Caucasian athletes also used approach/problem-focused coping significantly more than African Americans. There were no significant differences in any coping styles between Hispanics and Caucasians or between Hispanics and African Americans. With regard to gender, females used approach/problem-focused and avoidance/emotion-focused coping styles significantly more than males. In another study, Anshel, Kang, and Miesner (2010) sought to identify the coping styles of competitive athletes, using the Coping Style in Sport Inventory, as a function of gender (male and female) and race (African Americans and Caucasians) in response to stressful events experienced during competition. Males used more approach coping than females, and Caucasians used more approach coping 
than African Americans. Lastly, Puente-Díaz and Anshel (2005) examined the effect of culture on coping strategies, measured by the COPE Inventory (Carver et al., 1989), as well as the mediation of perceived controllability, measured by the Controllability Scale (Terry, 1991), between these two variables in Mexican and American tennis players. Mexican athletes identified "receiving negative comments and body language from coaches and relatives" and "experiencing an injury during a match" as the top two sources of stress during competition. American athletes cited "receiving negative comments and body language from coaches and relatives" and "opponents cheating" as the top sources of stress. Mexican athletes generally reported greater perceived controllability than their American counterparts. Furthermore, Mexican athletes reported higher levels of active coping, planning, positive interpretation and growth, and denial coping strategies. They found that perceived controllability mediated the relationship between culture and active coping. Also, perceived controllability partially mediated the relationship between culture and planning. Lastly, perceived controllability did not mediate the relationship between culture and positive interpretation and growth, focusing, venting, behavioral disengagement, and denial coping strategies.

Like Plaatjie and Potgieter's (2011) investigation, most of Anshel's studies dealt with acute stressors in sport rather than chronic stressors, such as long-term injuries. These studies also did not account for SES or social class. Moreover, the complexity of this topic may not have been able to be adequately captured using quantitative instruments. While these aforementioned studies demonstrated significant differences in coping styles among males and females and various ethnic groups, the authors failed to 
provide meaningful explanations to support their conclusions. Analogous to Thorpe's (2010) critique of traditional quantitative methodologies in studying extreme sports, these studies on coping may also reinforce stereotypes (e.g., females using primarily emotionfocused coping) while failing to account for the complex cultural dynamics, regional differences, and intricate political histories of the participants. Reducing the participants to homogenous groups (e.g., Australian males, Australian females, Caucasians, Mexicans, etc.) who perceive certain stressors similarly and employ identical coping strategies oversimplifies a very complicated issue. In other words, the predominant use of scales may marginalize the participants' subjective experiences. For these reasons, qualitative methodologies may provide a more comprehensive account of how an athlete's cultural context influences how he or she copes with injury and other chronic stressors.

\section{The Influence of SES on Stress and Coping}

To date, no research has examined the influence of SES on coping with athletic injury. Interestingly, individuals from lower SES backgrounds may be more likely to be injured. For instance, Shi et al. (2011) observed a significant relationship between low family income levels and non-fatal, unintentional injuries in Chinese undergraduate students; however, these injuries (e.g., falls, traffic injuries, animal/insect bites) were not limited to sport. Similarly, Potter et al. (2005) observed a significant inverse relationship between SES and non-fatal injuries among Canadian adolescents. No research has specifically looked at the relationship between SES and the prevalence of injuries in sport; however, the stress injury model, which posits that one's personality, history of 
stressors, and coping resources may indirectly play a role in sustaining an injury (Andersen \& Williams, 1988; Williams \& Andersen, 1998), would suggest that athletes from low SES backgrounds are more susceptible to injury. Particular stressors encountered by low SES individuals are discussed in more detail below.

Various studies in biology, public health, medicine, psychology, and sociology disciplines have consistently shown a clear relationship between SES and health, where lower SES individuals are more likely to have worse health outcomes (Chibnall, Tait, Andresen, \& Hadler, 2005; Jarvis \& Wardle, 1999; Kunst, Groenhof \& Andersen, 1999; Lin, 2011). Behavioral responses to stressful events largely contribute to this tendency. For example, low SES individuals have generally been found to smoke more (Jarvis \& Wardle, 1999), eat more unhealthy food (Mead, Gittelsohn, Roache, \& Sharma, 2010), and exhibit more sedentary lifestyles (Holt, Kingsley, \& Scherer, 2011; Wold \& Hendry, 1998). Parents from low-income families have reported that financial barriers and time management/scheduling issues (e.g., working multiple jobs) are major challenges to facilitating sport participation in their children (Holt et al., 2011). Other disparities include limited access to clean air and water, exposure to lead paint, social isolation, availability of public spaces (e.g., parks and recreation facilities), proximity to hospitals and other medical treatment facilities, and availability of health insurance, which Silk and Andrews (2012) attributed to the withdrawal of social welfare programs from the 1950s to the 1990s in the United States. Because of economic strain, exposure to violence, and frequent family transitions (e.g., divorce, changing homes, etc.), poverty is also associated with more family conflict (Santiago \& Wadsworth, 2009). Clearly, a 
deficiency in resources augments the stress experienced by populations disadvantaged by socioeconomic class. Nonetheless, even when opportunities and resources are available, Kristenson et al. (2004) noted that differences in stress levels and lifestyles still exist between high SES and low SES groups. These authors suggested that some low SES individuals acquire the belief that nothing they do can help or control their unfortunate circumstances, and they develop feelings of helplessness. The unique circumstances faced by people from various SES backgrounds may influence the coping styles they use in response to injury.

While no study has specifically examined the relationship between SES and coping with athletic injury, others have looked at the influence of SES on coping with other stressors. Ouwehand, de Ridder, and Bensing (2009) examined SES differences in middle-aged and older adults' use of proactive coping to prevent problems associated with aging and found that SES was positively correlated to proactive coping. The authors suggested that individuals from lower SES backgrounds may experience more persistent issues, such as chronic financial difficulties, which require greater attention and drain them of resources to proactively seek preventative health care. Additionally, Christensen, Schmidt, Hougaard, Kriegbaum, and Holstein (2006) looked at SES and coping patterns in 40 to 50 year old Danish individuals with musculoskeletal injuries. These authors found a significant positive relationship between problem-focused coping and social class, where women from higher social classes used more problem-focused coping. Also, avoidance coping was negatively related with social class, where men from lower social classes tended to employ more avoidance coping strategies. In another study that focused 
on women experiencing infertility-related stress, lower SES women used more passiveavoidant coping (Lykeridou et al., 2011). Other studies have revealed little or no differences in coping strategies as a function of SES. Chenube and Omumu (2011) examined the grief experiences and coping strategies in widows using the Widow Status Inventory developed by these authors. While lower SES widows reported more negative grief experiences than higher SES widows, there were no significant differences in coping strategies used.

The coping strategies employed by individuals to manage minor or major stressors carry significant implications for desirable outcomes and overall psychological well-being. For example, Iwasaki (2006) found that leisure coping buffered perceived stress and that this effect was substantially stronger for individuals from a lower social class regardless of gender or age. Using the COPE Inventory (Carver et al., 1989) to measure coping strategies of men diagnosed with HIV, Antoni et al. (1991) found that denial and behavioral disengagement predicted greater perceived stress. Furthermore, Antoni, Esterling, Lutgendorf, Fletcher, and Schneiderman (1995) found that denial and behavioral disengagement were associated with worse disease progression one year after the initial diagnosis. Similarly, Carver at al. (1993) looked at coping strategies in women diagnosed with breast cancer and found that both denial and behavioral disengagement were positively related to perceived stress, while planning negatively related to perceived stress. Using the COPE Inventory, Litman and Lunsford (2009) measured coping strategies in 451 individuals ranging from ages to 18 to 51 who had experienced varying types and degrees of stress in the past six months. They were asked 
questions regarding post-event well-being and health outcomes. The authors found that the use of planning positively predicted self-efficacy. Planning and positive reframing were positively related to improved personal growth (i.e., optimism, improved selfconcept). Venting and behavioral disengagement were both predictors of diminishment (i.e., reduced self-esteem, pessimism). Venting, behavioral disengagement, and selfdistraction were significantly related to post-event illness. Acceptance and the use of instrumental support predicted both positive and negative outcomes on well-being.

\section{Intersections among SES, Race, and Ethnicity}

Certainly, race and ethnicity cannot be overlooked when discussing issues pertaining to SES. Race refers to a socially constructed concept entailing fluid classifications that signify social conflicts and interests and infer meanings based upon perceived physical differences (Omi \& Winant, 1994). Racial groups are categorized by natural or assumed traits, as well as the meanings attributed to these traits. Research in the past century has shown that the concept of race is biologically invalid (Graves, 2002; Omi \& Winant, 1994). In other words, the various racial categorizations do not account for the variation in biological traits. Given that the concept of race is based on socially constructed ideas relating to human potential and abilities (Smedley \& Smedley, 2011), this is an inappropriate term to use when discussing variations in coping strategies. Nonetheless, prior research (e.g., McIlvane, 2006; Nixon, 1996) has used this term; thus, both race and ethnicity is used interchangeably depending on the particular study that is being addressed. For the purposes of this review, however, the term ethnicity is 
preferred. Ethnicity is defined as "a cultural heritage that people use to identify a particular population...based on cultural traditions and history" (Coakley, 2009, p. 276).

By and large, ethnicity is strongly related to SES in the United States. For example, $30 \%$ of Hispanics and 22\% of African Americans account for the more than 13 million families living below the poverty line (Simms, Fortuny, \& Henderson, 2009). As for educational attainment, the National Center for Education (2010) reported that over half of Hispanic family heads (i.e., parents or breadwinners of family) lack a high school diploma or equivalent, compared with one-fifth of Blacks, one-fifth of other non-Whites (e.g., Asians/Pacific Islanders), and one-sixth of Whites. Hispanics also have the highest high school dropout rate at 21\%, followed by Blacks (8\%), Asians/Pacific Islanders (6\%), and Whites (5\%). Furthermore, Hispanic fourth graders have the highest relative attendance of high-poverty schools at $52 \%$, followed closely by $47 \%$ of Blacks, as compared to $6 \%$ of Whites. Likewise, the proportion of fourth graders who qualify for free lunch programs include $77 \%$ of Hispanics, $74 \%$ of Blacks, $68 \%$ of Asians/Pacific Islanders, and $29 \%$ of Whites. With regards to occupational matters, unemployment rates of African Americans are double those of Whites (Rodgers, 2008). Moreover, African American men working full-time jobs earn $72 \%$ of what the average White male earns and $85 \%$ of the average White female's earnings. Evidently, SES issues cannot be ignored when discussing the significance of ethnicity and vise-versa.

These abovementioned socioeconomic disparities typically result in higher levels of stress for minority populations. Williams and Rucker (1996) noted that racial discrimination is an unfortunate aspect of social life which causes certain ethnic groups to 
be treated differently or unfairly. Traditional beliefs about racial groups are often subtly or overtly translated into policy decisions which limit the opportunities for certain groups and reflect unequal power differentials. Frequent contact with such barriers, obstacles, and indignities facilitates higher stress levels and consequently more negative psychophysiological consequences over time (Williams \& Collins, 1995). To illustrate this point, Schoendorf, Hogue, Kleinman, and Rowley (1993) found that infant mortality rates were significantly higher in offspring of college educated Black women compared to similarly educated White women. Other negative childhood outcomes, such as low birth weight, have been associated with mothers from lower SES and ethnic minority backgrounds, particularly Blacks and Hispanics (Fiscella, Franks, Gold, \& Clancy, 2008). Similarly, Mossakowski (2008) found that Black and Hispanic young adults reported significantly more symptoms of depression than Whites. Likewise, Brady and Matthews (2008) found that Black adolescents with few assets reported more negative life events than White adolescents with few assets. In addition to the adverse psychophysiological outcomes that result from stress, Fiscella et al. (2008) noted that an ample number of other health issues simply result from unequal access to quality care among different ethnic and SES groups.

While little research has looked the relationship between ethnicity and stress and coping in sport, research outside the sport domain has discussed how individuals from different ethnic backgrounds cope with negative life events. Conway, Magai, McPherson-Salandy, and Milano (2010) proposed that racial discrimination was a major stressor encountered by ethnic minority groups; therefore, the context of how they 
experience discrimination may influence the coping strategies they employ. With this concept in mind, Williams, Spencer, and Jackson (1990) found in several interviews that African American women predominantly expressed the use of passive coping in dealing with racial discrimination. This author attributed this to their low perception of control. Furthermore, Noh, Beiser, Kaspar, Hou, and Rummens (1999) interviewed refugees from Southeast Asia and found that passive coping was often used to deal with racial discrimination that they experienced while living in Canada. The authors concluded that this was a reflection of their collectivist cultural norms, where values such as avoidance, self-regulation, and interpersonal harmony are privileged over individualism and confrontation.

Farmer and Ferraro (2005) proposed the minority poverty hypothesis, which inferred that being a combination of both African American and low SES is associated with poorer health and psychological well-being, as well as having less coping resources or employing maladaptive forms of coping. Using the Vanderbilt Multidimensional Pain Coping Inventory (Smith, Wallston, Dwyer, \& Dowdy, 1997), McIlvane (2006) found that African American women dealing with arthritis pain used more religious coping, wishful thinking, seeking of social support, and emotional expression than Whites. This was even more pronounced in lower SES African American women. Furthermore, they found that African American women from lower SES backgrounds used more maladaptive cognitive appraisals and coping strategies, such as less positive appraisal, catastrophizing, and self-isolation. Also, Gary, Arango-Lasprilla, and Stevens (2009) looked at the physical and psychosocial outcomes, as well as the coping strategies used 
by patients with traumatic brain injury following surgery. They found that African Americans reported poorer physical function and psychosocial outcomes than their White counterparts. African Americans and Hispanics tended to used more emotion-focused coping, such as distancing and accepting responsibility than Whites. Contrary to these findings and the aforementioned theory, Borrill, Fox, and Roger (2011) found that among a sample of UK students who often engaged in self-harming activities (e.g., cutting), Blacks scored higher on a measure of problem-focused coping, and Asian students scored higher on avoidance coping. While there does appear to be some converging themes with regards to ethnicity and coping, there are many different factors (e.g., age, birthplace, residence, family size, nature of problem or stressor, etc.) that influence the use of particular coping strategies.

Other studies have looked at how Hispanic and/or Latino/a individuals have coped with immigrating to the United States. Employing semi-structured interviews, Shinnar (2008) examined how Mexican immigrants living in Nevada coped with negative self-identity brought on by racial discrimination. She found that some individuals moved away, or dissociated from the group, which she referred to as social mobility. More common, however, was the use of social creativity, which entailed efforts to achieve favorable group comparison to others, such as finding new characteristics unique to their particular in-group. Additionally, Pitale (2010) qualitatively examined how Hispanic adolescents who had moved to the United States coped with the difficulties of acculturation, particularly the distress of being separated from one's family, having trouble with a new language, and desiring more friendships. The most common coping 
strategies that these individuals employed were communication, learning and studying, self-talk, and thought-focused coping. As seen, both Shinnar (2008) and Pitale (2010) illustrated different ways of managing a dramatic life transition (i.e., immigrating to the United States).

While ample research has generally demonstrated that having a low SES background and/or being an ethnic minority increases one's risks of developing physical and psychological health problems (e.g., Brady \& Matthews, 2008; Chibnall et al., 2005; Farmer \& Ferraro, 2005; Fiscella et al., 2008; Gary et al., 2009; Holt et al., 2011; Jarvis \& Wardle, 1999; Kunst et al., 1999; Lin, 2011; McIlvane, 2009; Mead et al., 2010; Mossakowski, 2008; Santiago \& Wadsworth, 2009; Schoendorf et al., 1993; Williams \& Collins, 1995; Williams \& Rucker, 1996; Wold \& Hendry, 1998), other studies have revealed exceptions to this idea. One of the more well-known findings is the "Latino Paradox." Although Latinos generally have higher poverty rates, less education, and less health coverage than Whites, they generally have lower mortality rates (Eschbach, Ray, \& Markides, 2004), as well as less mortality rates caused by cardiovascular disease and cancer (Liao et al., 1998). Similarly, Cagney, Browning, and Wallace (2007) found that foreign-born Latinos had a lower prevalence of asthma and other respiratory issues compared to Whites and Blacks born in and outside of the United States, but this was only true for Latinos who lived in enclave neighborhoods where social networks are dense. The authors suggested that this structure makes it easier to share health information with each other. Additionally, Latinos have lower rates of low birth weight and infant mortality compared to Whites (James, 1993). Interestingly, other individuals 
who live in predominantly Latino communities may be able to reap these same health benefits. Relative to mothers who resided in Hispanic counties with less than a $1 \%$ Latino population, White and Black mothers who lived in counties with at least a 50\% Hispanic population have been shown to have a one third reduction in infant mortality, as well as a modest reduction in low birth weight and other risks of preterm delivery (Shaw \& Pickett, 2013). These authors concluded that certain aspects inherent within the Latino culture, namely family cohesion and social support, may buffer some of the health-related risks associated with being a racial minority and from a low SES childhood background. Additionally, they noted that the concept of motherhood is very important within the Latino culture; thus, they may be encouraged to adopt healthier behaviors, such as not smoking, during pregnancy relative to other cultural groups.

There is also some evidence of a similar paradox in Blacks or African-Americans. Although Blacks would be expected to have poor health outcomes, they have reported fewer mental disorders than Whites (Keyes, 2009; Williams \& Harris-Reid, 1999). Likewise, Blacks have also scored higher on several measures of flourishing (e.g., social coherence, social growth, environmental mastery, positive relations with others, purpose in life, etc.) than Whites, except for social acceptance, which is seen as a disadvantage of being Black due to perceived discrimination (Keyes, 2009). Controlling for perceived discrimination yielded higher scores on most measures of flourishing, or better mental health, in Blacks. The use of religion has been suggested as a mechanism which buffers some of the stressors experienced by Blacks. Attendance of religious services has typically been higher among Blacks than among Whites (Taylor, Chatters, Jayakody, \& 
Levin, 1996). Certainly, religious involvement has been shown to buffer the psychological distress experienced by Blacks as a result of racial discrimination (Ellison, Musick, \& Henderson, 2008). The use of religion has also been associated with higher levels of subjective well-being and lower incidences of mental health problems (Ellison, Boardman, Williams, \& Jackson, 2001).

Some low SES individuals have also defied their odds, exhibiting exceptional health. Dupre and George (2010) examined this phenomenon in a longitudinal study spanning from 1992-2008 with a cohort of 9,761 men and women who were between the ages of 51 and 61 at the beginning of the study. They found that males without a high school diploma were more likely to expect to live to the age of 85 years than males who had attained at least a high school education; although, individuals without a high school education had higher rates of depression, chronic disease, physical impairment, and other unfavorable health outcomes. Nonetheless, the authors found many protective mechanisms in individuals without a high school education that were correlated with exceptional health. In women, these included having a stable marriage, having a steady job, never smoking, being physically active, and having parents that lived to at least the age of 85 years. Protective mechanisms in men without high school diplomas were having no children, never smoking, and being physically active. Chen, Miller, Lachman, Gruenewald, and Seeman (2009) proposed that low SES individuals who demonstrate resilience adopt a "shift-and-persist" approach to life problems. People who utilize this strategy manage their stressors through cognitive reappraisals and emotional regulation (shift) and endure life with strength by focusing on the future (persist). These authors 
examined the relationship between the "persist-and-shift" strategy, measured by selected subscales from the Primary and Secondary Control questionnaire (Wrosch, Heckhausen, \& Lachman, 2000) and the Planning and Making Sense of the Past questionnaire (Prenda \& Lachman, 2001), and allostatic load, a calculated risk score based on seven different physiological systems, across varying levels of SES (defined as highest level of parental education attainment) in a sample of 1,207 adults with ages ranging 25 to 74 . They found that adults who grew up in low SES households and utilized "persist-and-shift" approaches had lower levels of allostatic load; whereas, this strategy was not found to have a protective effect in high SES individuals. Consequently, the ability to reframe a situation favorably and stay committed to one's goals enables low SES individuals to effectively cope with hardship. Similarly, in another study of 121 children aged nine to 18 who had been diagnosed with asthma, a "shift-and-persist" strategy was associated with less physiological inflammation from asthma, as well as less impairment (i.e., use of rescue inhalers and school absences due to asthma) in low SES children (Chen et al., 2011).

A hardiness personality may also help buffer some of the major stressors faced by low SES individuals. This personality construct entails having a sense of control, being committed to oneself and work, and viewing stressors as challenges instead of threats (Huang, 1995). Williams and Lawler (2001) examined the influence of hardiness dispositions, measured by the Dispositional Resilience scale, (Bartone, Ursano, Wright, \& Inhraham, 1989), on stress and illness in 100 women living at or below the poverty line. They found that hardiness moderated the stress-illness relationship, where women 
who were low in hardiness and had many perceived stressors had higher levels of illness. Additionally, White women with high amounts of perceived stress were more likely to have a greater number and severity of illnesses than high stress Black women. These authors concluded that even though living in impoverished conditions typically results in community stress, it may foster more hardy attitudes compared to individuals living in higher SES neighborhoods. Those with hardiness personalities tend to appraise events more accurately and cope more effectively. Consequently, growing up in low SES backgrounds may have some positive implications for stress and coping.

\section{The Role of SES and Ethnicity in Sport}

While the sport psychology literature has failed to address SES and other diversity issues, sport sociologists have extensively discussed the role of social class and ethnicity in sport. Sport sociology research has demonstrated how ethnic stereotypes are often a by-product of discrimination. For instance, coaches and other athletes often make comments reflecting stereotypes about black athletes' supernatural physical abilities (Harrison \& Lawrence, 2004; Lawrence, 2005). These attitudes in turn lead young Black athletes, particularly males, to believe that it is their biological and cultural destiny to be dominant athletes and that sport is the only avenue of gaining respect and achieving material success (Wenner, 1994). Consequently, these "positive" representations of Black athletes actually limit the social possibilities of this ethnic group. Although they are exposed to other aspects of their culture (e.g., language, literature, and art) at a young age, the realities of many young Black males have demonstrated that athletic merit drives the culture (Messner, 1992a). Another significant finding is that African Americans and 
Latinos disproportionately participate in less physical activity compared with Whites due to limited time and resources, which in turn is caused by poverty, segregation, and discrimination (Cutts, Darby, Boone, \& Brewis, 2009). Furthermore, although wellestablished athletes may be paid well, initial salaries for ethnic minorities tend to be inexplicably lower than their White counterparts. For example, some estimates have suggested that a Major League Baseball team can sign five baseball players from Latin America for the price of one U.S. born player (Coakley, 2009). Clearly, these intersections among SES, race, and ethnicity have profound implications in sport and exercise.

With these concerns in mind, the meanings of sport may vary depending on one's ethnic and socioeconomic background. For instance, Shinn (2002) contended that "Sport is a source of a new gendered pan-ethnic Latino/a pride, with cultural tradition, popular folklore, psychic and social connection to a distant homeland" (p. 240). In other words, sport is more than mere entertainment; beneath the surface, one can learn a great deal about the way a particular group constructs reality. The perception and performance of masculinity in sport can be interwoven into this discussion, as well. Laberge and Albert (1999) found that upper-class boys participated in sport in order to gain leadership skills, which was vital to their view of masculinity. On the other hand, working-class boys reported that sport provided a venue to gain social acceptance and show mental toughness. In regards to ethnicity, Klein (1995) suggested that Hispanic males are often stereotyped as having a need to dominate and control through sexuality, fighting, and other forms of aggressive and competitive behavior, a concept known as machismo. In 
contrast to this commonly held view, he observed that Hispanic baseball players exhibited more "tender emotions" in comparison to their White counterparts during an ethnographic study. These "tender emotions" included caring interactions with children and fans, expressions of vulnerability and feelings, and physical displays of affection. Hence, the cultural context of Hispanics may promote gentler and more compassionate forms of behavior.

Social class influences the economic implications in sport, as well. While there is limited research on the pressures to obtain and maintain athletic scholarships in athletes from low SES and/or ethnic minority backgrounds, the significance of athletic scholarships to White middle-to-upper class athletes has been addressed. For instance, Dyck (2006) noted that Canadian parents, who can afford to pay for their children's college education, invest a great deal of their time, money, and other resources to improving their kids' athletic skills in pursuit of athletic scholarships in the United States. Through ethnographic investigations, he found that Canadian athletes viewed a "full ride" scholarship as a sign of prestige, which reflected positively on their parents.

Furthermore, the authors found that many participants generally believed that American degrees are more highly regarded than Canadian degrees; although, no compelling evidence suggests this. Because of this, there is an underscoring belief that one is "set for life." Younger low SES athletes and their families may also view athletic scholarships as a sign of prestige; however, more importantly, athletic scholarships are also perceived to be essential for social mobility. Messner (1992b, 2007) stated that boys from lower class backgrounds are likely to make an investment in sport since other options of supporting 
their families are limited. There is more at stake for these individuals; therefore, they experience more pressure, which in turn leads to greater levels of stress. Often, these athletes lack the resources to train, hone their skills, and be noticed by others. Because athletic programs at colleges provide this support, there is arguably significant value in athletic scholarships granted to lower SES athletes.

Despite the widespread beliefs about the value of college athletic scholarships, there has been little research regarding the stress associated with attaining and maintaining an athletic scholarship. A serious injury may threaten an athlete's source of financial aid; therefore, this represents an ideal circumstance to investigate how athletes cope with severe stress. Other cultural factors may also influence the athlete's psychological response to injury. Mann and Kato (1996) have called attention to the methodological failures to include or represent minority groups in the general psychology research. With respect to counseling, Ivey, D’Andrea, Ivey, and Simek-Morgan (2001) stated:

It is particularly important for counselors and psychologists to examine closely how economic factors impact the psychological health and personal well-being of poor clients and to be mindful that traditional counseling theories were developed by middle-class individuals who did not give these issues enough weight ( $\mathrm{p}$. xviii).

Similarly, Ryba (2009) has challenged sport psychology professionals (including researchers, educators, and consultants) to be critically aware of the ethnocentrism present in mainstream sport psychology research. She noted that much of the current knowledge base was formed from observations and practice involving White, heterosexual, middle-to-upper class athletes. Treating these findings from Western 
culture as universal laws of human nature is not amenable to the experiences of various members of disenfranchised groups. Unfortunately, there has recently been a sparse amount of peer-reviewed sport psychology articles and presentations at professional sport psychology conferences (e.g., AASP) that discussed or even mentioned cultural diversity issues pertaining to gender, class, race/ethnicity, sexual orientation, disability, and other matters (Kamphoff et al., 2010; Ram et al., 2003). Because of this predicament, Fisher, Roper, and Butryn (2009) have advocated for incorporating cultural studies into sport psychology, also known as cultural sport psychology. This approach locates an athlete's identity within a web of power dynamics that advantages some individuals, while disadvantaging others. In other words, a larger emphasis is given to the athlete's psychosocial context. For instance, NCAA athletes from low SES and ethnic minority backgrounds who participate in revenue-producing sports may experience stressors that other athletes do not. Given these concerns, the purpose of this study was to examine low-to-middle SES athletes' experiences of coping with injury via qualitative interviews. 


\section{Chapter 3}

\section{METHODS}

\section{Measures}

Demographics. Participants completed demographic information (Appendix A) pertaining to their age, gender, sport, eligibility level (year) in their sport, date of injury, type of injury, and estimated recovery time (from date of injury to expected date of full recovery). The date of full recovery referred to when they expected to be training and/or competing at a full level again.

SES. In order to assess SES, participants completed the FAS II (Currie et al., 2004), which contains the following items: “(1) How many vehicles does your family own? (2) Growing up, did you have your own bedroom? (3) During the past 12 months, how many times did you travel away on vacation with your family? (4) How many computers does your family own?" Two modifications were made to the FAS II for this study. First, "Growing up," was added to the beginning of the second item in order to ensure the participants knew the question referred to where the majority of their childhoods were spent rather than the living quarters they may have been residing in temporarily as college students, such as dorms or off campus apartments. Second, the word "holiday" was changed to "vacation" in the fourth item to reflect American dialect. Scoring procedures can be found in Appendix B.

Boudreau and Poulin (2009) noted that household income is a sensitive issue; thus, when adolescents and younger adults are asked to report parental education, occupation, and income, there tend to be low response rates. Furthermore, younger 
individuals may not be knowledgeable about their household incomes. Likewise, for this study, college students may not have had this information available; thus, a simpler measure (i.e., questions adolescents and young adults could identify with more) was selected. Currie, Elton, Todd, and Platt (1997) demonstrated that response rates in adolescents increased when using the Family Affluence Scale I (FAS I), as compared to inquiring about father's occupation. The FAS I (Curie et al., 1997) is identical to the FAS II, except that the FAS I inquires about the number of telephones in the household rather than computers and does not include the item regarding family vacations.

The validity of the FAS I and FAS II has been well-established. Currie et al. (1997) found a strong positive relationship between FAS I scores and classification of father's occupation. Boudreau and Poulin (2009) also found that there was a significant positive relationship between FAS II scores and mother's occupation and highest level of education. Boyce, Torsheim, Currie, and Zambon (2006) found that FAS II scores significantly related to family income levels. Lastly, Lin (2011) found moderate associations between FAS II scores and education and occupation of both parents.

For the purposes of this study, scores were divided into the following groups: low SES (0-3), middle SES (4-5), and high SES (6-9). Other researchers (Boyce et al., 2006; Cho \& Khang, 2010) have recommended similar categorizations to interpret FAS II scores. The main purpose of the FAS II was to identify low-to-middle SES athletes for the interviews, or the second phase of the study.

Experiences of coping with injury. According to Nagy Hesse-Biber and Leavy (2011), exploratory studies, or investigations on underresearched groups or phenomena, 
are suitable for qualitative designs. Given that low-to-middle athletes' experiences of coping with injury have not been addressed, this study served to develop an initial understanding of this issue. This study was situated within a social constructivism paradigm, which emphasizes participants' subjective meanings of their experiences that are formed through interaction with others and through historical and critical norms that operate in their lives (Creswell, 2007). Accordingly, an inductive approach was utilized in order to interpret the participants' lived experiences.

Semi-structured interviews were used to examine low-to-middle SES athletes' experiences of coping with injury. The interview guide is presented in Appendix D. A semi-structured interview allows the researcher to ask specific questions in a systematic fashion related to the research question; however, the interviewee is allowed to digress (Berg, 2004). In doing so, the participant may shed light on a particular aspect of a phenomenon that the researcher had not previously considered. This allows the interviewer to probe beyond the predetermined questions. Generally, the interviewer seeks to understand the participant's point of view by asking open-ended questions. Through broad and open-ended questioning, participants are allowed to construct the meaning of the situation (Creswell, 2007).

\section{Participants}

The first phase of the study, where participants completed demographic information and the FAS II (Currie et al., 2004), was comprised of 34 (22 male, 12 female) NCAA Division I athletes at a university in the Western United States who had sustained an injury that: (a) occurred within a year of IRB approval, and (b) lasted (or 
was expected to last) four weeks until full participation in practice and/or competition. With regards to race/ethnicity, the sample consisted of athletes who identified as White or Caucasian $(n=16)$, Black or African American $(n=10)$, Asian American or Pacific Islander $(n=3)$, Latino or Hispanic $(n=3)$, and biracial $(n=2)$. The participants' sports included football $(n=14)$, gymnastics $(n=3)$, tennis $(n=1)$, soccer $(n=8)$, softball $(n=$ 1), baseball $(n=3)$, basketball $(n=3)$, and water polo $(n=1)$. Athletes had sustained injuries involving the knee $(n=9)$, shoulder $(n=7)$, hand and wrist $(n=3)$, foot and ankle $(n=3)$, elbow $(n=2)$, hip $(n=2)$, upper leg $(n=2)$, lower leg $(n=2)$, head $(n=3)$, and respiratory tract $(n=1)$. The mean recovery time (or estimated recovery time), which was defined as the amount of time missed from full participation in practice and/or competition, was 22.8 weeks ( $S D=15.6$ weeks) and ranged from 4 to 52 weeks. From this larger sample, the mean FAS II score was $6.03(S D=1.82)$.

Based on their FAS II scores, 11 participants (nine male, two female) were selected to take part in the second phase of the study, which entailed the interviews. This number of participants was approximately consistent with recent studies that have qualitatively examined injured athletes' coping strategies (Heijne, Axelsson, Werner, \& Biguet, 2008; Galli \& Reel, 2012a, 2012b; Podlog \& Eklund, 2006; Tracey, 2003; Wadey, Evans, Hanton, \& Neil, 2012). This smaller sample consisted of the following self-identified racial/ethnic breakdown: White or Caucasian $(n=2)$, Black or African American $(n=6)$, Asian American or Pacific Islander $(n=2)$, and Hispanic $(n=1)$. They participated in football $(n=8)$, gymnastics $(n=1)$, tennis $(n=1)$, and soccer $(n=$ 1). At the time of the study, 10 of the 11 participants had "full ride" athletic scholarships 
that provided compensation for $100 \%$ of their tuition and fees, and one participant had $35 \%$ of his education-related costs covered by an athletic scholarship. They had sustained injuries to the knee $(n=2)$, shoulder $(n=3)$, hand and wrist $(n=2)$, foot and ankle $(n=2)$, hip $(n=1)$, and upper leg $(n=1)$. Within this smaller sample, the recovery time $(M=19.5$ weeks, $S D=11.0$ weeks $)$ ranged from 4 to 39 weeks. More specific information about their respective injuries can be found in Appendix E. The mean FAS II score was $4.27(S D=0.90)$.

Purposive sampling methods were utilized to obtain 11 participants who: 1) were a member of an NCAA Division I athletic team; 2) sustained injuries within a year of IRB approval which lasted at least four weeks until returning to play; and 3) came from low SES or middle SES backgrounds. Six athletic trainers at a Division I institution were contacted in order to help identify and recruit participants who met these first two criteria.

Participant selection was delimited to college athletes as a matter of convenience sampling and to focus on one particular level of sport. For example, a college athlete may experience different stressors or appraise the same stressors in a dissimilar manner compared to a professional, high school, or middle school athlete. More specifically, pressures to retain an athletic scholarship may be intertwined within one's SES and cultural backgrounds, as well as the injury process. Because scholarship availability differs among NCAA Division I, Division II, and Division III institutions, where Division I schools typically possess the most scholarship funds, this study was delimited to Division I athletes. 
Participants were also required to have been injured for at least four weeks, or have an expected recovery time of at least four weeks. A significant, or long-term, injury presents a stressful and challenging circumstance for the injured athlete (Quinn \& Fallon, 1999). For example, an individual who is injured for six months is likely to cope differently than an athlete who is injured for three days. Consequently, this study was delimited to those athletes who were unable to train at a full level or compete for at least four weeks, or approximately one month. This study was also delimited to athletes who sustained their injury within a year of IRB approval so that athletes could recall detailed and accurate descriptions of their experiences. To this point, Nicholls and Polman (2007) have called for minimizing the time delay between the stressful event and the recall of this stressful event when utilizing retrospective research designs that measure coping in sport.

This study was further delimited to low-to-middle SES athletes in order to provide more meaning to a particular group rather than decipher the potentially different meanings between low SES and high SES individuals. In other words, the purpose of this study was to describe one aspect of a phenomenon among a targeted group rather than compare and contrast coping styles among different populations. Participants who scored between " 0 " and "5" on the Family Affluence Scale II (FAS II; Currie et al., 2004) were considered low-to-middle SES and eligible for this study.

\section{Procedure}

Approval was obtained from both the IRB (Appendix G) and the Head Athletic Trainer at a NCAA Division I university in the Western United States. Demographic 
items and the FAS II were combined into one questionnaire. Informed consent forms (Appendix C) and questionnaires were given to six athletic trainers, who distributed them to athletes in their respective sports that they supervised. After athletes signed the informed consent forms, they completed the questionnaires. This information was kept confidentially in a secure place. Athletes who scored in the low SES range (0 to 3) or middle SES range (4 to 5) on the FAS II were contacted via phone or email and asked to participate in an interview.

Prior to data collection, a pilot interview was conducted with a former NCAA Division I athlete who previously sustained a long-term injury in order to test and refine the interview guide. In the second phase of the study, the 11 selected participants took part in semi-structured interviews. The interviewee was given the opportunity to decide where and when the interview took place. As Seidman (1991) noted, allowing the participant to choose the interview site demonstrates sensitivity to potential power imbalances between the researcher and participant. Prior to the interview, participants were reminded that the purpose of the study was to examine the influence of SES on coping with athletic injury. I also briefly described my background with athletic injuries in order to build rapport with the participants. The participants were asked about their family backgrounds, stressors, reactions to their injuries, and coping strategies. The interview guide is presented in Appendix D. After the interview, participants were given an opportunity to ask any other questions, as well as provide feedback on the types of questions they were asked. The lengths of the interviews ranged from 30 to 70 minutes, 
and they were audio recorded with an Olympus ${ }^{\circledR}$ VN-5200PC digital voice recorder and transcribed verbatim.

\section{Data Analysis}

Key themes were identified within each interview and across interviews using an unstructured interpretational analysis (Côté, Salmela, Baria, \& Russell, 1993). This form of data analysis allows themes to emerge from the data rather than match participants' statements to a predetermined model. In other words, a classification system was developed inductively to specifically fit the interviewees' statements in this study.

Each interview transcript was read thoroughly multiple times in order to become well-acquainted with the data and capture the participants' meanings from their subjective viewpoints. First, raw data themes were formed from the participants' statements that pertained to the interests of the study, namely their socioeconomic backgrounds, perceptions of injury, emotional reactions, and coping strategies. Other substantial themes that recurred throughout the interview were coded, as well. Data saturation occurred at the eleventh interview. Second, after the raw data themes were fully extrapolated, higher order themes were created to reflect commonalities among the data both within individual transcripts and across multiple transcripts. The creation of higher order themes was complete when all raw data themes were able to be appropriately categorized without the creation of new higher order themes. Third, these higher order themes were further organized into general dimensions. Each raw data theme was closely scrutinized to assess its best fitting higher order theme and associated 
general dimension. As a result, the organization of the data was repeatedly modified until a final classification system was produced.

\section{Academic Rigor}

In qualitative research, it is critical for the researcher to acknowledge his or her biases and assumptions in order to gain an awareness of how they may impact the research process (Creswell, 2007; Seidman, 1991). Creswell (2007) stated:

Researchers recognize that their own background shapes their interpretation, and they 'position themselves' in the research to acknowledge how their interpretation flows from their own personal, cultural, and historical experiences. Thus the researchers make an interpretation of what they find, an interpretation shaped by their own experiences and background (p. 21).

Miyata and Kai (2009) suggested such self-reflexive practices as a means of enhancing the confirmability (i.e., an awareness of the influences between the researcher and participants) of the study. As a former NCAA Division I cross-country and track and field runner, I suffered several injuries, including two chronic injuries, femoral stress fractures and plantar fasciitis. Both of these injuries sidelined me for several months. Before I received an athletic scholarship as an upperclassman, I was originally a walk-on athlete striving to prove that I deserved athletic money. My desire to attain an athletic scholarship, compounded with my running injuries, led to a great deal of stress. While I did not come from a lower SES background, I was cognizant of how resources and connections played a role in my injury and rehabilitation. Because cross-country and track and field were not marquee sports, the athletic department did not provide coverage all of my necessary medical expenses. As a result, my teammates and I had to seek out other medical practitioners and pay for most of our health expenses. Some of my 
teammates were frequently able to access other rehabilitation services, such as massage therapy or physical therapy; however, I was unable to afford such luxuries that may have facilitated a quicker recovery. I was anxious to prove my worth to my coaches and teammates; however, because I was not as privileged as some of my teammates, I felt helpless at times. Given my athletic background, I was greatly interested in the coping strategies athletes used in response to being injured. Furthermore, in the present investigation, I hoped to capture the experiences of individuals who perhaps had much more at stake (i.e., a greater need for scholarship money) than I did, especially since their stress may have been exacerbated by hardship and other difficulties pertaining to SES. However, given that my knowledge of low SES individuals in relation to stress and coping was formed more through the lens of a researcher than from experience, I originally assumed that many individuals from low SES backgrounds would discuss bleak and depressing events. Through the process of interviewing the participants and analyzing the data, I developed a critical awareness of these generalizations that I initially carried as a researcher. Also, as an American from mixed ethnic backgrounds (fifth generation Mexican-American and fourth generation Western European-American), I may have been less aware of values and customs inherent to other ethnic groups, particularly individuals whose families immigrated to the United States more recently. Prior to data collection, I participated in a bracketing interview conducted by an experienced interviewer. In a bracketing interview, the researcher explores his or her biases and presuppositions that may influence how the study is designed and implemented (Dale, 1996, 2000). While bias cannot be completely eliminated, these 
predispositions are brought to the conscious awareness of the researcher. The bracketing interview consisted of my thoughts pertaining to my experience as a Division I athlete, my SES and family background, prior injuries, coping strategies that I employed, and other issues that were relevant to this study. While I discussed class issues relating to my sport, I was only able to make a limited amount of connections between SES and how I coped with my injuries. Nonetheless, this self-reflexive process revealed that I still possessed an assumption that SES would play a more profound role on how athletes from lower-income backgrounds coped with their injuries. In other words, I presumed that low SES individuals would discuss several negative life experiences, which would influence how they coped with injury. However, through my interactions with the participants, I recognized this was a flawed assumption.

Member checking, which entails sending the verbatim transcript to the participant, was used to enhance the credibility of the study per the recommendations of Miyata and Kai (2009). This practice allowed the participants to provide feedback on the meanings derived from the interview, and they also had the option of removing or elaborating on any of their statements (Dearnley, 2005). On an ethical level, member checking also narrows the power differential between the researcher and participant by facilitating a collaborative relationship. No significant changes were made to the transcripts. One participant requested the removal of "filler" language, such as "um," "uh," "like," and other vernacular. One participant also preferred the removal of one sentence that she thought may have portrayed her negatively. This request was honored, especially since it did not relate to the purpose of the study. Lastly, one participant clarified the meaning of 
one brief statement that appeared to be vague to her. Generally, there was minimal feedback from the participants.

Also, a reflexive journal was maintained throughout the data collection process. This contained an ongoing record of my thoughts, experiences, reactions, and any biases and assumptions that developed throughout the research process. Like the bracketing interview, a reflexive journal allows the researcher to examine his or her value-laden selfunderstandings and how they relate to the study (Morrow, 2005). This also contributed to the confirmability (i.e., the idea that the findings will represent the participants' experiences rather than the beliefs or biases of the researcher) of the study (Miyata \& Kai, 2009).

Lastly, a peer debriefing session was conducted with an experienced qualitative researcher in order to ensure the dependability of the findings, or the stability of the observations (Miyata \& Kai, 2009). 


\section{Chapter 4}

\section{RESULTS}

Each participant was assigned a pseudonym. More information regarding their demographics and injuries can be found in Appendix E. Thematic analysis of the data produced 73 raw data themes. They were organized into 16 higher order themes, which in turn were categorized under five general dimensions: cognitive appraisals, emotional reactions, problem-focused coping, emotion-focused coping, and avoidance coping. The full results of the content analysis are depicted in Appendix F.

\section{Cognitive Appraisals}

In order to investigate how athletes coped, it was essential to examine the underlying perceived stress associated with their injuries. The participants discussed their cognitive appraisals (i.e., initial thoughts and interpretations), which indicated that they perceived their athletic injury as a stressor. This general dimension consisted of two higher order themes: confusion and catastrophization.

Confusion. Several participants indicated that they slowly processed the events of their injuries. Accordingly, some athletes mentioned they were not immediately aware that they became injured. Catherine, who ruptured her Achilles tendon when performing a flip in practice, initially had no inkling that she had sustained an injury. She said:

I feel like I went into shock or something it was just like I was confused. I didn't know what had happened and...honestly didn't think it hurt that much like when it first happened probably because I had adrenaline. I was just tumbling, and I didn't know what was going on. It was just like my foot was just like hanging there. 
She expressed that she was in a state of shock and confusion, which contributed to the initial stress she experienced. She noted that it took her a few days (when she had surgery) to grasp the magnitude of her injury. Similarly, Ian mentioned that his injury "was a blur" when he tore his labrum, and he initially did not comprehend that he was injured. Furthermore, many participants were unaware of the seriousness of their injuries. Russell, Brett, Catherine, Emily, and Tony all mentioned that their recovery times were all longer than they had originally anticipated. In some cases, these perceptions influenced certain athletes' decisions to play through their injuries, which is discussed in more detail below under the higher order theme of avoidance coping.

Catastrophization. Several participants shared many catastrophizing thoughts in response to their injuries. Catastrophization refers to the act of having an irrational belief that is characterized by framing an event much worse than it actually is and/or expecting the worst possible outcome (Ellis, 1994). Many athletes predicted catastrophic consequences as a result of their injuries. For example, Sam's initial reaction to injuring his knee was that he was never going to play again. This injury caused him to miss the spring season. Although the spring football season was not as significant as the fall season, he noted that coaches made playing time decisions based on offseason performance. Since his injury occurred during a coaching transition, he assumed that because the coaches did not get a chance to see him perform, he would never play again. Likewise, Michael's initial reaction to being injured was that he would never start again: "I was like, 'Oh, no...dang! I'm starting. Now I end up not starting.' So that's the only thing I thought of." Similarly, after tearing his labrum, Ian believed he would never be 
able to use his shoulder the same way he did before his injury. Additionally, a few participants stated that injuries always happened to them, or happened to them more so than other people. For example, Russell's initial thoughts were "Man! Why is this happening?...Man, why me? Why does this kind of stuff happen to me sometimes?" In this particular case, Russell clearly experienced some self-victimization or a sense of unfairness. Such irrational thoughts were voiced by other participants, including Sam, Andy, and Ian. Many of these athletes expressed initial thoughts of helplessness in their susceptibility to getting injured.

In the context of Sam's family background, he particularly perceived his injury as a severely negative life event. As mentioned, his parents had separated, and his mother was recovering from brain surgery; thus, he was already experiencing ample stress at home. Additionally, he ended his relationship with his girlfriend during this time. For Sam, this long-term injury was yet another negative event that initially caused him to feel a sense of helplessness. He stated:

It's like what do I have going on for me, you know what I mean?...Usually, it's my football, but I don't have football, you know what I mean, and then nothing was going on good at home. It's like I don't have anything like-what positive things have happened, you know what I mean? Nothing.

Because of several events that occurred beyond his sport, he initially viewed his injury as evidence that nothing positive would happen in his life. In other words, because of recent events in his life, he assumed the worst possible scenario would occur. Overall, a common trend was that some of the participants initially interpreted their injuries in absolute terms (e.g., "never," "always," "nothing," etc.). 


\section{Emotional Reactions}

Participants identified some key emotional reactions, which influenced how they coped with their respective injuries. Three higher order themes emerged within this general dimension: sadness, frustration, and relief.

Sadness. Many participants expressed sadness upon realizing they were injured; however, the degree and time at which this occurred varied across participants. For example, Russell said he was sad for a couple days; whereas, Sam discussed being depressed for a month. On the other hand, Ian said his sadness did not sink in until he was at the hospital preparing for his surgery to repair his torn labrum. Andy did not report any sadness until after his surgery to fix the femoral acetabular impingement (FAI) in his hip. For Tony, his season-ending injury (broken foot) signified a missed opportunity to prove his worth to his coaches. He said:

I actually felt like crying at the time. It was like my freshmen season, like I wanted to prove myself not only to the players but like to the coaches because they pay me to play here, and I wanted to make it known that their money is not going to waste....I was the youngest player on the team...so I had felt like I had a lot to prove.

For several participants, sadness occurred due to the season-ending nature of their injuries. The timing and degree of sadness that the participants experienced had implications for how they coped, which is discussed more below.

Frustration. Frustration was a key emotion experienced by several participants for multiple reasons. Like sadness, this was also manifested to different degrees. Most of the frustration appeared to stem from a decrease in physical function as a result of injury. For instance, Catherine discussed her frustration with the long recovery time: 
When I finally got out of the boot...I felt weak, and like I never felt like that before. I'm like, "This is so weird," you know... and I saw that my calf had like no muscle, and I was just like, "What? Oh, my gosh!" It was like so quick how fast it lost that muscle, and I had to gain it all back, and I was just like, "This is going to take forever," you know? It was just frustrating.

Catherine had already been upset with her lengthy recovery time; however, after she noticed her calf atrophy, she realized she was going to have to put in more time to regain strength in that muscle. Similarly, Brett, who tore the triangular fibrocartilage complex (TFCC) in his wrist, was frustrated when he was unable to dribble a basketball, which was one of his post-surgery rehabilitation exercises. Because he was originally a basketball player in high school, he felt discouraged that he could not carry out a simple physical task that he was acclimated to doing on a frequent basis. Most of Andy's frustration also occurred post-surgery. He said:

I was frustrated because of the crutches...not being able to put pressure on it for a week was just so frustrating....I mean you can't even put on your sock. You can barely shower. Um, and it was just really frustrating not being able to do that and not being able to put pressure on it and uh-that kind of stuff....You can't poop like-you're just mad because like right after surgery, I couldn't pee.

Initially, Andy and other participants did not account for the temporary lack of physical ability associated with their recoveries; thus, they became irritated when they were forced to deal with these momentary setbacks. In a similar manner, Tony reported being frustrated at not being able to engage in any physical activity, such as running or swimming, in the early stages of his recovery due to his broken foot. All in all, these participants became frustrated that they momentarily lost the ability to perform basic physical activities which they had taken for granted. 
Relief. While the initial emotional reactions were mostly negatively toned, some participants actually expressed some relief throughout the injury process. Andy, in particular, said he felt relieved when he was diagnosed with FAI in his hip. Unlike other participants' injuries, Andy had previously been dealing with vague chronic pain in his hip for months. As he said, "It was kind of like exciting to me, which just sounds bad, but to understand it's not just in your head; you're actually injured." Considering that his surgery took place in the offseason, Andy was relieved to undergo a procedure that would supposedly eradicate his chronic pain, allowing him to play better once the regular season began. Additionally, while sadness and frustration were the salient feelings that Tony experienced in response to his injury, he did admit feeling somewhat relieved. As a freshman athlete, he was acclimating to having longer and more practices relative to his prior training experiences. Since he was physically tired, he welcomed some time off; however, as previously mentioned, his recovery time was longer than he originally anticipated. Although no other athletes expressed relief upon being diagnosed with their respective injuries, other participants, such as Catherine, indicated a sense of relief after their surgeries took place.

\section{Problem-focused Coping}

The problem-focused general dimension yielded five higher order themes: comparison to past experiences, commitment to rehabilitation, perseverance, tangible support, and informational support.

Comparison to past experiences. Many participants looked at past experiences to help cope with the stress of their present injuries. In many cases, this entailed 
participants comparing their current injury to past injuries. For instance, Richard, who tore the anterior cruciate ligament (ACL) in his knee, had sustained the same injury while he was in high school. While other participants discussed some confusion regarding the type or severity of their respective injuries, Richard immediately knew that he had torn his ACL since he was familiar with the physical sensations. Regarding lessons learned from his past injury, he stated:

I've learned...through past experience because the first time I was one of those guys that was just bitter about everything just like, "Aw, why me? Why me?"...just blamed the world. But this time it happened, I just like-I just didn't take it personal. I was just like, "All right this happened to me. I been through this before. This time, let's just, you know, have a fast recovery, think positively about it, and better things will come."

As seen, he mentioned having catastrophizing thoughts and other irrational beliefs in response to his injury that he sustained during high school; however, he deemed that this had not aided his recovery. Richard noted he was having a faster recovery than his prior ACL injury, which he partially attributed to learning lessons from his past experience. Other participants who mentioned catastrophizing when they initially appraised their injuries still managed to engage in positive thoughts to some extent at later phases in rehabilitation; however, Richard was able to immediately focus his efforts on getting healthy rather than dwell on his injury.

Simon, who suffered a torn ulnar collateral ligament (UCL) on his thumb, also talked about his past injuries. While this was his first time having surgery due to an athletic injury, he had learned from past experiences to not dwell excessively on the injury. Furthermore, he compared his injury to his past life experiences beyond his sport. For instance, he noted that having to deal with his family's house catching on fire or the 
gang activity at his high school presented a lot more challenges than his present injury; thus, he was prepared to manage this setback. He stated:

I've been through a lot of things in my life, so compared to having a surgery, which I've never had before, but dealing with things that I've done in my past, such as, you know, my house catching on fire, or just uh, other...daily stressors that we've talked about. It's not, uh, you know, not something that, you know, is too major. I was definitively able to-I've definitely prepared for anything to happen because I've done a lot of struggle in my life.

In Simon's case, this injury paled in comparison to the challenges and hardship that he faced growing up. Similarly, Michael mentioned the challenges of his socioeconomic background when discussing how he coped with his present injury: "[The injury] wasn't really a big deal to me. I'm able to look back at what I've been through knowing that I don't want to go back there." When Michael looked back on the adversity he had experienced growing up, such as the loss of friends due to gang activity, he was able to put his injury in perspective. In other words, he did not interpret this setback as a severely negative life event.

Commitment to rehabilitation. All participants mentioned a strong commitment to recovering. Almost no one indicated any issues with adhering to rehabilitation. To this point, Simon said, "If you really want to get back and you want to get healthy, you do whatever the trainer says....I want to get back as soon as possible, so I do whatever they tell me to do." Initially, Emily, who suffered a strained hamstring, was not as receptive to her rehabilitation protocol as other participants; although, she eventually compromised on a plan with her athletic trainer within a week of her injury. Like others, Emily mentioned that she was devoted to doing everything to compete again. Accordingly, in addition to completing her rehabilitation exercises, she did her own 
research on her injury to make sure the trainers were not omitting anything from her treatment and that she was doing everything within her control to recover in a timely manner.

Some participants drew connections between their family background and their commitment to rehabilitation. For example, Brett said consistency was a significant value that his father instilled in him. He stated:

When I couldn't dribble the basketball, or when I had different things like it was kind of hard to do rehab-like there was some days, you know, I would be like, "Man I'm sore, I don't want to-why is it so sore? I worked it out a lot yesterday," but just my dad has always been about being consistent and making sure that you like show up somewhere on time or make sure that when you go there you like work as hard as you can or what not so...that helped me out a lot....He called me every day, and...he was like, "Yeah, you up for rehab? Are you going?"... and you know he helped me out with being consistent.

Brett's father taught him to approach commitments with his best effort; hence, consistency was essential to putting forth his best effort in football, as well as recovering from his injury. Additionally, Tony discussed his strong determination to recover fully and be able to compete again. Not only was he committed to his rehabilitation exercises, but he also admitted to frequently nagging his athletic trainer to see if there was any extra work that could be done to maintain his fitness or help heal his foot faster. He said:

I was always on [my trainer's] a** and like "[Shannon] (athletic trainer's pseudonym), when can I do this? [Shannon], when can I start running?" because sitting on my butt...like you obviously lose shape, you gain weight, you lose muscle...like all that stuff, and I just didn't want to be there.

Tony mentioned that he did not want to feel useless, and he wanted to maintain his fitness as much as possible in order to compete well upon fully recovering. His trainer Shannon often had to hold him back from doing too much weight-bearing exercise too soon, but he 
exhibited a strong willingness to do any alternative forms of physical activity, such as swimming, which indicated his strong resolve to return to play. Tony credited this determination and work ethic to his father, who worked long hours to support his family. Growing up, he was accustomed to his father getting up early in the morning to go to work and coming home late in the evening without complaining. Tony stated that other people he knew would have had a lazier approach and consequently experience negative outcomes, such as weight gain; however, he believed he was fortunate to have parents that modeled strong work ethics.

Perseverance. Several participants discussed adopting attitudes resembling perseverance in managing their injuries. The connection between family background/SES and coping was most evident in this higher-order theme. Ian noted that he was taught to never give up when "times get hard." Ian admired his mother as a single parent, who worked extra hours and days in order to make ends meet for both of them. He applied this same approach to his injury. Emily also credited her parents with a "never give up" attitude, or perseverance, in both her sport and injury. Additionally, Michael mentioned the importance of not giving up on his injury and sport. When asked how his family background and SES influenced how he coped with his injury, he replied:

That goes back to like never giving up and stuff like that "cause I could have just said in my head like, "What if?"...like just gave up-“I'm gonna lose my spot," whatever. And then-and then thinking about what I wanted-what I want to do, and I know I don't want to go through that again, I was not. So keeping my head up because it was easy-it would be easy to just put my head down and just give up on everything... and then end up being back—-back on the streets....

Michael viewed his sport as a way out of an undesirable life and an opportunity to get an education. As he mentioned, if he had given up on his sport due to injury, he would have 
lost more than just a starting position. Eliminating football also meant losing his education, which he and his parents valued. Generally, his background profoundly influenced him, as he demonstrated resilience in response to his injury, which was not as dire as other circumstances, such as gang activities and drive-by shootings that he had endured in his past. Sam also exhibited a great deal of perseverance. After being depressed initially, Sam noted that he eventually showed more resilience later in his recovery. He stated:

I can't give up on it because I just have one chance to do it. It's just four years, you know what I mean?...Can't give up on it....My dreams, and you know I have to get my degree, and I can't just give up so...that's what drives me...to make like my mom happy pretty much....to be like so she feels I'm successful pretty much.

As mentioned, Sam's mother was recovering from a serious illness. His mother was his motivation to focus and persevere through the challenges, including both his injury and family problems. Like Michael, Sam also mentioned the need to stay focused on getting his college degree. He inferred that his college education would ultimately take him far and better his life.

Tangible support. Multiple participants mentioned several instances where they sought physical support from others. For example, Brett noted that his girlfriend and mother would visit and prepare dinner for him since this was difficult to perform with an injured wrist. Also, both Ian and Michael, who had shoulder injuries, had loved ones (girlfriend and brother respectively) who assisted them in putting on their shirts when they visited. Andy, who had very limited hip motion after his surgery, also valued his mother's help. He said: 
I couldn't even [put on my clothes]. I'm like "Sorry Mom, I'm 21," but uh, I would sit on the couch, and she would fill up my water bottle every time it was empty because I was supposed to be drinking a lot of water and she'd make me food...I'd just sit on the couch...um, she just basically did everything for me.

Andy mentioned that he was fortunate to have a close-knit family, especially his mother, who could afford to visit him. Catherine also mentioned that her father assisted with physical support, such as driving her to her doctor's appointments since her torn Achilles prevented her from driving. Unlike most of the other participants, Tony mentioned that his school did not pay for all of his medical bills; therefore, his parents had to financially assist him. He stated that even though they had little extra money, his parents paid his bills without complaining. As mentioned previously, he said his parents had made sacrifices in order to allow him to play soccer at a high level.

Informational support. In addition to their discussions with athletic trainers, some participants indicated that other people provided them with information about their injuries. For example, Emily mentioned that her parents did their own research about her injury. Likewise, Catherine sought advice from a teammate who previously had also ruptured her Achilles tendon. Her teammate confirmed that it would approximately be a six month recovery. She also advised Catherine to go to a physical therapist in order to regain strength function completely. Finally, she warned her about common mistakes to avoid that might hinder her recovery. Additionally, Catherine received ample emotional support from her teammate, which is discussed below.

\section{Emotion-focused coping}

The emotion-focused coping general dimension comprised three higher order themes: positive reframing, use of religion, and emotional support. 
Positive reframing. Several participants focused on positive thoughts at various phases in their rehabilitation in order to abate the negatively toned emotions (e.g., sadness, frustration) that they experienced. While Sam initially reported some catastrophizing thoughts, he tried to adopt a more optimistic attitude towards his injury. He did this by reducing the perceived magnitude of his injury (i.e., telling himself that his injury was not so bad), as well as focusing on positive aspects of his life. For example, he reminded himself that he was fortunate to not have to pay tuition. As a freshman, Sam also noted that he had ample time remaining in his collegiate career to compete and accomplish his goals. He said:

You know one thing that like drives me through the injuries [is]...that I have a lot of time left, that I have my school paid for, and that I could, you know, bounce back, and that it's just all in my head, you know what I mean? That it's not as bad as it seems. That's what helps drive me to like keep going.

Likewise, Ian focused on being grateful that he was getting his education, which he said would benefit him in the long run. Generally, he tried to focus on the positive things he had in his life rather than dwelling on what he did not have, an attitude which he attributed to his mother. Similarly, Russell and Richard were appreciative of the fact that they did not have to pay for their medical bills, which they both said would have made their respective recoveries more stressful.

Furthermore, four participants mentioned that they found upsides to their injury. For example, Russell indicated that this injury would prepare him for future injuries or other forms of adversity:

Well, it definitely taught me a lesson. You know, going through an injury, it could take you on a high, or it can take you on a low. Basically, like imagine just a highway, and it just splits off into two, and you can get bummed out about it and 
be like, "Aw man, why did this happen to me? Man, I just don't even want to play football" or whatever the sport may be anymore because this stuff happens. Or you can go the other way and be like, "You know what? This can be a setback, but it's only going to make me stronger." I mean, you probably heard that before. What doesn't kill you makes you stronger. That's exactly the truth. So it really motivated me, so even if I do get a future injury in-hopefully not in the near future-I know how to exactly deal with it, and, you know, try to follow the same thing I did last time.

Unlike other participants, such as Richard, Russell did not draw upon lessons from a prior injury; however, through positive reframing, he was able to find a way that this injury may benefit him in the future. Additionally, Brett was able to glean positive value from his injury. Having injured the wrist of his dominant hand, he was in a cast for two months. While he acknowledged that it was tough to write, shower, and perform other simple activities, he was appreciative of the fact that he learned how to perform tasks, such as writing, with his non-dominant hand. He mentioned that he was still able to do these tasks at the time of the interview, which took place approximately six months since his full recovery and about nine months since he had his cast removed; hence, this was a long-term benefit that he enjoyed. Similarly, Ian, who tore his labrum, was unable to lift weights using his upper body; however, he was able to focus on his lower body. He said:

I think it's kind of a blessing in disguise...because, um, like I've always had a really strong upper body, but my lower body has been my weak part, so...[I'm] forced to work on my legs, my calves, and just let this rest, and then once I come back, I'll be more explosive.

Like Russell, Ian implied that he would be a stronger and better player when he returned to play. In all of these cases, participants coped with their injuries by focusing on positive aspects that they perceived were balancing out the downsides of their respective injuries. 
Use of religion. As seen, many participants came from families with strong religious backgrounds; thus, prayer and spirituality played a significant role in coping with their injuries. For instance, Simon affirmed that his core background was based on a belief and faith in God; therefore, he continued to trust that a higher power would help get him through his injury. In other words, he implied that his belief in God would help him through a fast recovery, and if not, then a greater plan existed for him. Additionally, Catherine mentioned that prayer was a primary means of coping with her injury. Because of her faith in God, she was confident that "everything had a way of working itself out." Prayer also played a profound role in the emotional support that she received from family and friends, which is discussed below.

Furthermore, Sam mentioned that one of the more significant events that helped him cope with his injury was that he rekindled his relationship with God. As mentioned, he did not want to talk to his mother about the injury because he did not want to risk worrying her. Furthermore, he received negative feedback from his father or girlfriend when he tried to talk to them; hence, he was unable to rely on them for support.

Consequently, he turned to God to help cope with his injury. He said:

Like seriously, my biggest thing that [happened].... 'Cause first I used to be a really good Christian and stuff, and then I like kind of fell off, and then through being injured this last time, I started being real close to God again. And like my whole motivation now is actually making me a lot more optimistic, and it's to not worry about anything like in the future.

Because of this setback, his religious and spiritual identities became more salient to him. He attributed his positive thoughts and optimism to his renewed relationship with God. 
Emotional support. Most participants acquired various degrees of social support, principally emotional support, from family members and other significant individuals in their lives. Many participants discussed the encouragement that they received from various people. For example, Catherine said that some of her extended family members, who had been injured previously, reassured her that she would get through her injury. Likewise, she became closer with a teammate, who had sustained the same injury. Her friend was also a devout Christian, and they often prayed together. She stated:

I also had a teammate last year that she tore her Achilles also, so she helped me a lot with it, and she is also Christian, and like we both shared that similarity, and we were really close like last year, and she helped me so much through it, and she told me what to expect and how I'm going to feel, but you're just going to have to deal with it and like it's part of God's plan, you know, like don't go against it.

While other participants mentioned that they did everything within their control to expedite their rehabilitation, Catherine was receptive to her friend's feedback that her injury was outside of her control and attributed it to a plan initiated by a greater power (i.e., God). Like Catherine, Andy also received ample prayers from family members who were hoping his surgery would be successful, which helped him cope with his injury. Likewise, Brett said his grandmother frequently called him to inquire about his status and offer her prayers. More generally, both Andy and Brett noted the emotional support that he received from his family was essential to his recovery. Richard also mentioned support from siblings, particularly his older brother, who had also played football. Richard said:

My family is very supportive. Like that's what really kept me going. My older brother just-he is one motivator, I'll tell you that much....Like he-he's one of 
those brothers who are just like talking to you constantly. Like he wants to know, you know, what's going on, like is everything okay, like he's just really caring and just loving, and I know he just really cares for all of us. Like he really stepped up to the big brother role and just taking charge so...he's just good at setting a good example and, I'm just trying to follow.

As stated previously, Richard and his siblings moved in together after his parents separated in order to maintain the closeness of their family. Clearly, the motivation from his brother in addition to the other support that he received from family helped Richard cope with his long-term injury. Tony shared these thoughts, as well. Initially, his instincts were to go to his family for support. He said:

When I was hurt, like the first thing was that some people would go to their friends or go to their coaches or go to other people. My first instincts was to go back to my parents like they raised me. I would be able to tell them everything and like how I felt and as soon as I got hurt, and I told them that I want to go home, and I told them that I just want to be with you guys, and even though I wasn't doing like anything there-I was just like sitting down and watching TVthere was just like the feeling being home is a lot different from being in my dorm room. Just sitting there, like I just felt a lot more alone.

Growing up, Richard had been taught to value his family and to always stay loyal to them. Clearly, this was influential in his decision to seek and accept emotional support from these individuals. Spending time with his family helped alleviate his sadness and frustration. While he appreciated the positive feedback from his friends and coaches, he noted that the "warmth" of his family could not match the other sources of social support.

\section{Avoidance Coping}

The avoidance coping general dimension consisted of three higher order themes: playing through pain, isolation, and mental distraction.

Playing through pain. Initially, several participants chose to ignore the pain and play through their injuries. Sam, who tore his meniscus, continued practicing on his 
injured knee for several weeks. Not only did he exacerbate his injury, but he also developed a cyst on his knee as a result of playing through it. He said:

At first when I felt it, I was like, "Ah, my knee sore. I can deal with it," you know what I mean? And eventually throughout the season-when the season started, it started getting worse and worse to the point where I couldn't do anything....There is times where I would go to practice and I would have to run, like I'll have to do a play, because I was trying to stay out as much as I can. I'll tell everyone I can't do it, but they didn't understand that, and I should have been real serious with them, but I kind of like thought I could fight through it, but that was stupid because it just got worse and worse.

While there may have been some pressures to play injured, Sam generally assigned himself the blame for underestimating the seriousness of his injury and believing he could ignore his injury. On the other hand, Brett experienced direct pressure to play with his injured wrist. He said:

I hurt it on probably like the last thirty seconds of the game, and we were in a close game. So when it got hit, my coach called a timeout, and I went to the sideline, and I was like, "Man, my wrist really hurts," and he was like, "We need you! It's the last couple seconds of the game!" And you know, they tell you to be mentally focused and tough and things like that. So basically the very next play, I went back out there and kind of just forgot about the pain for the play for the last couple seconds of the game.

Brett played through his injury for the duration of the regular season before he got surgery after the season. As seen, he coped with his injury by ignoring his pain in order to fulfill his playing obligations to his teammates and coaches. Emily also tried to continue playing through her pain, but after one week, she realized her injury (hamstring strain) was more serious than she had initially judged. Additionally, Tony tried to play soccer on a fractured foot; like Sam, however, he made his injury worse. He also believed he had an obligation to help his teammates. Initially, Tony convinced himself that he only had a bruised foot, and he believed he had a high tolerance for pain. Even 
after an official diagnosis was made, he often tried to negotiate shorter recovery times with his trainer. For example, he tried walking immediately after surgery, but he quickly found that this was not possible. In these aforementioned cases, the participants ignored their pain as much as possible and perhaps denied the realities of their respective injuries.

Isolation. While many participants sought social support at various points in their recoveries, some athletes tried to distance themselves from others. As mentioned previously, Sam had learned that he could not rely on his father for support due to the negative feedback he had received every time he suffered a setback in his sport. At first, he did not want to make his mother worried, so he avoided discussing his injury with her, as well. Accordingly, he mentioned that he "shut himself off" from others, particularly his teammates because he did not want to talk about his injury. Similarly, Brett originally avoided discussing his injury and surgery with his parents and teammates because he did not want them to worry. Also, Emily expressed aggravation when her teammates asked about her injury; hence, she tried to avoid speaking to her teammates. Like others, Tony also closed himself off from his teammates. He said:

As soon as...I knew I was going to be hurt for a while, there was a good time I was reserved. They would be like, "Dude, are you okay?" and I'm just like "Yeah," and I would just close my door, and I would just like sit there.

On the other hand, Tony did not feign interaction with his family. As mentioned previously, his first inclination was to seek the support of his family. Overall, some athletes isolated themselves from others, particularly teammates, in order to avoid thinking about their injuries. 
Mental distraction. Some participants engaged in activities that helped keep their minds off of their injuries. Although Brett admitted that he initially wanted to keep to himself post-surgery, he went to the movies with his friends, which he appreciated. Later in his recovery, Brett and his teammates also went swimming during their preseason training camp, which helped keep his mind off of his injured wrist. He said:

When I was hurt, I had teammates around me that just kind of - they didn't even realize...it was, you know, kind of helping me out because I wasn't thinking about it. But then if you don't have that, what activity do you do? Do you just sit there and kind of, you know, dwell on the injury, or you realize a lot more about the pain that you have, or that's when you start taking more drugs for the pain and you know probably because like me...I still have my pain medication.... I didn't take that much of it. I was actually able to be okay..... when someone else started to like get my mind distracted or something like that, it's like, "Oh, okay. I don't remember the pain anymore."

Clearly, in addition to being supportive, Brett's teammates also distracted his mind from the pain and other stressors associated with his injury. He also mentioned that his girlfriend and family helped him keep his mind off the injury when they visited him. Additionally, Tony turned to lifting weights in order to prevent himself from dwelling on his injury. As mentioned, he was actively seeking any physical activity that his athletic trainer allowed. When he was approved to lift weights, he mentioned that this helped keep his mind off the negative aspects of his injury. 


\section{Chapter 5}

\section{DISCUSSION}

This study qualitatively examined the experiences of lower-to-middle class Division I athletes coping with injury. The participants discussed their cognitive appraisals, emotional reactions, problem-focused coping strategies, emotion-focused coping strategies, and avoidance coping strategies regarding their recent injuries. This was the first investigation to look at the coping strategies employed by injured athletes through the lens of their socioeconomic backgrounds and other related identities. The results of this study indicated that SES is a relevant consideration when examining an athlete's responses to injury; however, as the integrated sport injury model (WieseBjornstal et al., 1998) suggests, there are several other personal and situational factors to consider. Rather than isolating SES as an independent variable, the semi-structured interview format allowed the participants to discuss other factors (e.g., religion, cultural background, family values) that were related to SES. An additional strength of this study was that it made several contributions to both the mainstream and cultural sport psychology literatures. While participants were unable to draw many connections between their SES backgrounds and coping strategies that they employed during their respective injuries, there were still many noteworthy findings.

First, this study provided support for Lazarus' (2000) assertion that positively toned emotions can result from stress. For example, Andy, who had been dealing with vague chronic pain for over six months, reported feeling relieved after he was diagnosed with his injury. Despite the four to six month recovery ahead of him, he was relieved to 
have an official diagnosis because his athletic trainers and other personnel could devise a specific rehabilitation protocol that would allow him to play pain-free again. Similarly, some athletes, such as Catherine, indicated relief after surgery in spite of the pain and discomfort because they were making progress in returning to competition. Also, Tony was relieved that he would be able to take some time off. Even though he was primarily frustrated as a result of his injury, he noted that he had been physically exhausted from the increased training volume that he was acclimating to as a freshman; thus, he was somewhat amicable to some time away from his sport. Generally, these findings reinforced Lazarus' claim that stress and emotions are experienced simultaneously. Furthermore, both positive and negative emotions can arise concurrently as a result of a perceived stressful event.

Second, this study also extended some support from previous findings on the timing and nature of avoidance coping. This investigation was consistent with Albinson and Petrie's (2003) findings that avoidance coping strategies were predominantly used in the early stages of their injuries (i.e., within one week of injury) in athletes that sustained long-term injuries (i.e., four weeks or more). For instance, both Sam and Tony distanced themselves from their friends and teammates within the first few weeks of when their injuries occurred. Additionally, several athletes ignored the initial symptoms of pain and tried to play their injuries. There were two possible reasons that may have explained why avoidance coping was largely used in the earlier phases of injury. First, in some instances, athletes may not have fully comprehended and accepted the consequences of their injuries, which influenced their decisions to play through pain. Second, if they were 
aware of the long-term nature of their injury, they may have felt overwhelmed, as they perceived that a lengthy rehabilitation would tax their physical and emotional resources. Consequently, some participants may not have been as motivated to address the injury directly (i.e., employ problem-focused coping) in the earlier phases of injury. Nonetheless, this study showed that all forms of avoidance coping are not necessarily maladaptive. To this point, many participants reported using mental distraction techniques that helped them manage their injury and rehabilitation. For instance, Tony credited other forms of physical activity, namely lifting weights, with preventing him from dwelling on his injury. Contrary to Roth and Cohen's (1986) assertion that avoidance coping is preferable when emotional support is limited, many participants suggested that their family and friends played a significant role in offering social support, as well as aiding them in mental distraction. For example, Brett mentioned that swimming with his friends helped keep his mind off of his injury. In agreement with the findings of this study, Carson and Polman (2010) noted that some forms of avoidance coping (e.g., thought stopping, cognitive distraction, physical distraction) play a facilitative role in managing short-term mood states as a result of athletic injuries. Furthermore, these authors suggested that some of these avoidance coping strategies can result in many long-term benefits, such as personal performance development, time management, planning for the future, undertaking community development work, pursuing media/business interests, and others.

Third, there was a strong connection between participants' family backgrounds and the use of religion, or prayer. Seven of the 11 participants stated that religion was an 
integral part of their identities and/or essential to coping with their respective injuries. The practice of religion may be more prevalent in sport. In support of this view, Storch, Roberti, Bravata, and Storch (2004) found that athletes at an NCAA Division I school in the Southeastern United States reported higher levels of religiosity than non-athlete college students at the same institution. On the other hand, Bell, Johnson, and Petersen (2009) found that non-athletes at an NCAA Division III religious practice institution reported higher degrees of religiosity than athletes; while, they observed no significant differences between athletes and non-athletes at an NCAA Division III non-religious practice institution. Consequently, Division I athletes may utilize prayer and other forms of religion or spirituality more frequently than other college athletes; however, since there appears to be several factors involved in this phenomenon, no firm conclusions can be established. More generally, in a random sample of U.S. adults, Baker (2008) found that women, African Americans, and those with lower incomes pray more than men, Whites, and those with higher incomes. While it may be too large of a generalization to attribute the religiosity of the participants in the present study to these sociodemographic variables, it is worth noting that more than half of the sample identified as Black or African American. Religion was salient in four of the six Black or African American participants' experiences of coping with injury. Furthermore, as per the delimitations of the study, all of the participants were from low-to-middle SES backgrounds. Gallup and Lindsay (1999) mentioned that those with higher incomes are typically less concerned with appealing to supernatural powers in order to advance their social standing as opposed to those with average or below average incomes. The findings were also 
consistent with the work of Czech, Wrisberg, Fisher, Thompson, and Hayes (2004), who found that Christian Division I athletes often used prayer to reduce stress and provide a sense of security.

Fourth, some participants mentioned wresting positive value from their injuries, a major finding that is consistent with previous studies (Gould et al., 1997; Galli \& Reel, 2012a, 2012b; Podlog \& Eklund, 2006; Udry et al., 1997; Wadey et al., 2012). For instance, despite the physical debilitation of their injuries, some athletes mentioned they were able to improve on some of their skills and abilities pertinent to their sport, such as increasing lower body strength, becoming ambidextrous, and enhancing strategic awareness by spending more time watching film. Similarly, Udry et al. (1997) found that physical skill development occurred in several injured Olympic United States skiers, while Wadey et al. (2012) found that injured athletes increased their tactical and technical awareness. Some athletes, such as Russell and Richard, also reported that they formed positive expectations of the future, which supported the findings of Gould et al. (1997). Additionally, Galli and Reel (2012a) found that athletes reported stress-related growth during their injuries through a number of mechanisms, such as increased spirituality and interpersonal connectedness. The present study supported some of these previous findings. For example, Sam reported that he "found God again" and reignited his relationship with God. As mentioned, several other participants indicated that religion and prayer played a role on how they coped with injury; however, it was unclear, except in one case, whether they became more spiritual during rehabilitation. Regarding interpersonal connections, Catherine also discussed how she became closer with one of 
her teammates, who had previously sustained the same injury. Brett also developed closer relationships with his teammates, an unexpected significant source of social support. While sport psychology professionals, coaches, athletic trainers, and other individuals working with athletes should encourage positive reframing, Udry (1999) warned that it often takes time for an athlete to wrest positive value from his or her injury, as was the case in this study. Accordingly, most participants did not realize these lessons until the middle to the end of their recovery periods.

Fifth, one of the more remarkable findings was the self-reported perseverance in relation to how athletes approached their injuries. In other studies, this phenomenon has also been referred to as determination/motivation (Gould et al., 1997), resilience (Galli \& Reel, 2012a), and fighting spirit (Augutis \& Anderson, 2012). Generally, these constructs refer to very similar coping strategies; hence, researchers may want to consolidate these terms into one theoretical category. With regard to the present study, the connections between SES and coping were more evident in this higher-order theme. For instance, Ian credited his perseverance to his mother, who dealt with adversity when he was younger. For Michael, he simply did not want to return to his old life, which influenced him to be perseverant. In other words, he viewed sport as a way out of an undesirable living situation. Additionally, Sam expressed his motivation to not give up in order to make his mother, who was recovering from a serious illness, proud. This study was in line with other investigations outside of sport (e.g., Massad et al., 2009) that showed that exposure to adversity does not necessarily reduce resilience. In some cases, being exposed to adversity may prepare an individual for an event that is perceived as 
less stressful. Little research has been conducted on how athletes from low SES backgrounds handle adversity in sport. A caveat to these findings is that Division I athletes, who habitually manage the time, pain, and fatigue of practice and preparation, must exhibit a high level of commitment in order to achieve top performance (Lazarus, 2000). Consequently, regardless of SES, perseverance may be more common in high performing athletes than novice athletes or non-athletes.

Positive reframing and perseverance were two of the more common coping strategies that emerged from the participants' accounts. Taken together, this was indicative of a "shift-and-persist" strategy (i.e., reappraising a negative event and remaining committed in the face of adversity), which has been shown to buffer stress in low SES individuals (Chen et al., 2009). While some participants initially reported catastrophizing thoughts, many of them exhibited this "shift-and-persist" approach by engaging in cognitive reappraisal, or positive reframing, and exhibiting perseverance. For example, Sam discussed several of his initial thoughts, such as his belief that there was nothing positive in his life and that he would never play again. Later, however, he began to focus on positive thoughts, such as being grateful that his tuition and other expenses were being paid for, as well as adopting an optimistic attitude towards his progress (shift). He also affirmed that he could not give up and that he had to be strong for his family in order to make them proud (persist). As mentioned, the family backgrounds of the participants may have influenced these coping strategies (i.e., positive reframing and perseverance) that they employed. 
While the findings of the present study and other inquiries (Chen et al., 2009; Chen et al., 2011; Massad et al., 2009; Williams \& Lawler, 2011) demonstrated successful coping despite unfavorable odds, it is important not to glorify the struggles of low SES individuals and other disadvantaged populations. These results could easily be misinterpreted, where one might subscribe to the notion that anyone can be successful in spite of an underprivileged upbringing. Certainly, the austere realities (e.g., lack of access to affordable health care, employment uncertainty, dismal living conditions, exposure to crime, family conflict, etc.) faced by low SES individuals cannot be overlooked. Glossing over these unfortunate facts only serves to reinforce class hegemony. To this point, Ryba (2009) has argued that the ethnocentric bias of mainstream sport psychology research may emphasize the overdetermination of individuals. Theories derived from White middle-upper-class individuals do not reflect the realities, or psychosocial contexts, that are representative of all individuals. Perhaps this study is one example of the larger scale tensions between the sport psychology and sport sociology disciplines. While sport psychology professionals have generally supported practices consistent with positive psychology, sport sociologists have expressed caution that such research may unintentionally reinforce social inequalities. In order to mend this gap, some researchers (e.g., Fisher et al., 2009; Ryba, 2009) have advocated for a cultural sport psychology perspective, a newer paradigm which recognizes constructions and locations of identity, particularly how they progress within fluctuating contexts of power relations. 
Delimiting the study to Division I athletes provided a good opportunity to examine the potential pressures faced by athletes to keep their athletic scholarships. Quite surprisingly, however, most of the participants did not believe that losing their scholarships was a remote possibility. In particular, most of the football athletes believed that their scholarships could only be terminated for conduct reasons. Encouragingly, although Brett did not say he was worried about his own scholarship, he commented that anyone could find a reason to take away a scholarship from an athlete. From a sociological perspective, this indicated that they were unaware of the power imbalances that exist between NCAA Division I athletic departments and their athletes. For example, Beamon (2008) noted that when their athletic roles are emphasized over their academic roles, student-athletes often feel exploited and unprepared for careers. This demonstrates that college athletes need to be educated about their susceptibility to being exploited by the NCAA, athletic departments, and other governing forces in sport. On a related note, many participants mentioned that they played through pain due to pressures from coaches. Accordingly, this study provided some evidence for Nixon's (1992) theory of risk and "sportsnets," where people in higher authority (i.e., coaches) minimize their risk (i.e., outcome of game, job security) by transferring this risk (i.e., injury) to their athletes. Because many athletes express a desire to play through their injuries, society may fail to critically analyze the potential power imbalances and how they contribute to the exploitation of athletes, particularly those in Division I settings and revenue-producing sports. Since an athlete receives a scholarship and other benefits, he or she may feel obligated to comply with the coach's wishes. More research is needed on 
the influence of SES/social class on athletes' decisions to be complicit in playing through pain and injury.

\section{Limitations}

One limitation of this study was that the participants were at various stages in their rehabilitation when they were interviewed. Accordingly, the interviews were retrospective; hence, the participant may not have been willing or able to articulate critical information that addressed the research question. As a consequence of both of these limitations, participants may not have been able to remember aspects of the coping process that were salient at earlier phases in their injuries. Future research should include data collection at multiple points in an athlete's rehabilitation like prior studies (e.g., Albinson \& Petrie, 2003; Carson \& Polman, 2008, 2010; Quinn \& Fallon, 1998, 2000) have done, and these stages should be clearly defined.

A second limitation concerned the use of NCAA Division I athletes. Attending a four year college or university (even with a scholarship) typically requires a great deal of resources; hence, this may explain why it was difficult to find participants from low SES backgrounds. As seen, only three individuals scored in the low SES range (0-3) on the FAS II, and no one scored below a "3" on this scale. Also, the tangible support structure in place at this Division I institution may have buffered some of the stress experienced by the participants. For example, athletic departments at Division I schools typically pay for some or all of the health care services required for athletes to recover from sport-related injuries. They also provide other resources (e.g., whirlpool, ultrasound, laser therapy, etc.) and personnel (e.g., athletic trainers) to ensure both an effective and efficient 
rehabilitation. Even if an athlete and his/her family lack resources, the athletic department is usually able to compensate. Accordingly, some participants, such as Russell and Richard, mentioned that their injuries would have been substantially more stressful if they were not provided with such resources.

Another limitation of this study was the severe gender imbalance, as only two females participated in this study compared to nine males. Perhaps male participants may not have been willing to discuss their emotions and how they coped with injury. Sharing such experiences may be emasculating (Vishnevsky, Cann, Calhoun, Tedeschi, \& Demakis, 2010), particularly in a "hypermasculine" sport culture. There was not as large of a gender imbalance in the sample of participants who completed the first phase of the study (22 males, 12 females). Putting this into perspective, approximately $59 \%$ of injured male athletes scored in the low or middle SES range on the FAS II; whereas, only about $17 \%$ of injured female athletes scored in the low or middle SES group. While young males view sport as a means of providing a better life for themselves and their families (Messner, 1992b, 2007), females may be discouraged from making the same decisions. This may be due to the fact that there are less high-paying jobs in women's professional sports. Nonetheless, there are theoretically an equal amount of male and female scholarships in NCAA Division I athletics; thus, there does not appear to be a clear reason for why there were few females in the low-to-middle SES range. Moreover, one of the two females in this study originated from a European country, which may differ in some aspects of sport culture, such as attitudes towards female participation in sport. 


\section{Future Directions}

As mentioned, participants were largely unable or unwilling to discuss issues of SES or social class, particularly as it related to their experiences of coping with injury. This may have been a result of social norms where discussions of SES or social class are taboo (Sanders \& Mahalingam, 2012). Also, participants may not have been educated about issues pertaining to SES or social class; therefore, they were not able to articulate how their own SES or family background had an impact on their experiences of coping with their injuries. In fact, one participant candidly admitted to his lack of knowledge on the topic. The difficulty in getting people to talk about SES may illustrate why this topic has never been examined in the sport psychology literature. In spite of this difficulty, this issue needs to continue to be investigated. As Sanders and Mahalingam noted, classbased privileges are supported and reproduced by the (lack of) discourse on SES and social class; hence, this is not an issue we should turn our backs on. More nuanced methodologies should be employed in order to bring these issues to the forefront. First, focus groups would be recommended for future studies. Such a format would allow participants to feed off of each other when discussing their experiences. In other words, hearing another participant's stories may conjure up memories of a similar event that would not have otherwise been salient during an interview. Likewise, participants may feel more comfortable discussing their SES backgrounds in a group where many individuals may have been dealing with similar circumstances. Second, from a quantitative perspective, a larger and more comprehensive scale measuring SES should be developed that corresponds to modern technology (e.g., the use of smart phones) and 
encompasses multiple factors, such as income, assets, resources, number of parents, parental occupation(s), parental education, and neighborhood environment. The fouritem FAS II used in the present study may not have accurately represented the participants' SES. Third, utilizing mixed methodologies may enable researchers to gain a better grasp of this issue. For example, using questionnaires, such as the COPE Inventory (Carver et al., 1989) or Coping Inventory for Stressful Situations (Endler \& Parker, 1990), in conjunction with follow-up interviews may add more depth. A questionnaire can serve to illuminate the use of coping strategies that were not salient to the participants, and follow-up interviews can provide the participants an opportunity to identify other coping strategies that they used and/or provide more meaning to their experiences. Other measures, such as keeping a diary on one's thoughts, influential emotions, and coping strategies, can also serve to triangulate the data.

As previously stated, several participants engaged in both positive reframing and perseverance, which resembled a "shift and persist" strategy (Chen et al., 2009). To date, the "shift-and-persist" strategy has not specifically been examined in sport contexts. Given the numerous challenges and threats that high performing athletes must manage (Lazarus, 2000), this would be a great venue to study this phenomenon, particularly as it relates to low SES individuals coping with chronic stressors, such as long-term injuries. More broadly, the "shift-and-persist" strategy should also be investigated with respect to other individuals disadvantaged by gender, race/ethnicity, sexual orientation, disability, and other identities as a mechanism of overcoming adverse outcomes. 
As mentioned, each participant was interviewed at a different stage in his or her recovery, and these interviews were retrospective. In order to address these limitations, future research should include data collection at multiple stages in an athlete's rehabilitation like prior studies (e.g., Albinson \& Petrie, 2003; Carson \& Polman, 2008, 2010; Quinn \& Fallon, 1998, 2000) have done, and these phases should be clearly demarcated.

Another suggestion for future research would be to conduct a similar study on high school athletes. Colleges, particularly Division I institutions, may buffer some of the financial stress associated with an injury by paying for the athlete's medical expenses and providing access to rehabilitation services via athletic trainers and medical practitioners. On the contrary, high schools typically do not provide as numerous resources as most colleges; athletes and their families are burdened with medical expenses and other costs. Moreover, athletes who are trying to continue their careers in college have more at stake if they have not received scholarship offers yet. An injury could hinder one's chances of earning an athletic scholarship, which potentially has more significant implications for low SES individuals. In fact, while the focus was on Richard's present injury, he did mention that other schools withdrew their scholarship offers when he tore his ACL in high school. Consequently, conducting a similar study with high school athletes may be a good avenue to advance research in this area.

Given that SES is intertwined with other elements of one's identity (e.g., race, ethnicity, gender, and religion), these complex intersections should continue to be investigated. Of particular interest are the experiences of female athletes from low SES 
backgrounds. To date, little is known about how gender and class identities interact to influence behavior in sport. Some research has addressed the meaning of sport in young males from low SES backgrounds (Messner, 1992b, 2007); however, no studies have examined whether low SES families view sport as a viable way out of poverty for females. Consequently, future studies that situate females' experiences of coping with injury and other stressors within the context of their SES backgrounds and gender identities would be meaningful.

A minor theme not related to the purpose of the study that emerged was the fear of re-injury. A few athletes said they were nervous about returning to practice and/or competition after recovering. For example, Emily stated that she felt several vague pains in her hamstrings, causing her to be uneasy when returning to play. Also, after Tony's broken foot healed, he mentioned he did not feel normal during his first few practices. Recently, some researchers (e.g., Heijne et al., 2008; Podlog \& Dionigi, 2010; Podlog \& Eklund, 2006) have begun to look more closely at the experiences of athletes returning to sport following serious injury. More research is needed in this area in order to construct theoretical models pertaining to this phenomenon. The influence of cultural factors, such as gender, ethnicity, and SES, on athletes' experiences of returning to sport after injury needs to be investigated, as well.

\section{Conclusions and Implications}

This study presented some implications for sport psychology professionals, coaches, athletic trainers, and other personnel; however, it is worth mentioning that these findings should not be generalized to all low-to-middle SES college athletes. As the 
integrated sport injury model (Wiese-Bjornstal et al., 1998) posits, there are a wide array of other factors in addition to SES and family background, such as gender, ethnicity, athletic identity, prior injury experience, playing status, time in season, and other variables that influence how an athlete responds to injury. Methodologically, reducing one or more of these factors to independent variables in order to examine their effects on coping is unhelpful given the complex interaction of factors that determine an athlete's identity, as well as how he or she will respond to a particular situation. As McGannon and Johnson (2009) noted, because some forms of data analysis, such as analyses of variance, are strengthened by the assumption that within-group variance is minimized, relying solely on such techniques may be counterproductive to accounting for the many intricacies in identity categories. Accordingly, since this study was situated within a social constructivism paradigm, the aim of this study was to become familiar with the participants' subjective experiences, as well as the meanings they derived from them (Creswell, 2007).

Consequently, individuals who work with injured athletes should consider other events beyond sport which may be impacting how the injured athlete is managing the injury. They should also assist the athlete in mobilizing his or her social support network. For example, a sport psychology professional can reach out to the coach, teammates, family, and other individuals who are willing to provide genuine and meaningful support. This may include the use of prayer and other forms of coping pertaining to religion if it entails a significant part of the injured athlete's identity. Athletic personnel must understand that low SES individuals may need more social 
support (i.e., tangible, informational, or emotional support) depending on one's family background. Nevertheless, one must exercise caution in forcing too much social support on the injured athletes. Sometimes, an injured athlete needs time away from teammates (i.e., avoidance coping) to prevent to him or herself from dwelling on the injury. Likewise, sport psychology professionals and other individuals should attempt to facilitate the adoption of perseverance in injured athletes. One may look at an athlete's psychosocial context in order to identify prior instances where he or she persevered or showed resilience. Generally, coaches, athletic trainers, sport psychology professionals, and other individuals should be more conscious of SES and other elements of one's identity when collaborating with injured athletes. 


\section{References}

Albinson, C., \& Petrie, T. (2003). Cognitive appraisals, stress, and coping: Preinjury and postinjury factors influencing psychological adjustment to sport injury. Journal of Sport Rehabilitation, 12, 306-322.

Amirkhan, J., \& Auyeung, B. (2007). Coping with stress across the lifespan: Absolute vs. relative changes in strategy. Journal of Applied Developmental Psychology, 28, 298-317.

Andersen, M. B., \& Williams, J. M. (1988). A model of stress and athletic injury: Prediction and prevention. Journal of Sport and Exercise Psychology, 10, 294306.

Anshel, M. H., Kang, M., \& Miesner, M. (2010). The approach-avoidance framework for identifying athletes' coping style as a function of gender and race. Scandinavian Journal of Psychology, 51, 341-349. doi:10.1111/j.1467-9450.2009.00796.x

Anshel, M. H., Sutarso, T., \& Jubenville, C. (2009). Racial and gender differences on sources of acute stress and coping style among competitive athletes. The Journal of Social Psychology, 149, 159-177.

Anshel, M. H., Williams, L. R. T., \& Hodge, K. (1999). Cross-cultural and gender differences on coping style in sport. International Journal of Sport Psychology, $28,141-156$.

Antoni, M. H., Esterling, B., Lutgendorf, S., Fletcher, M. A., \& Schneiderman, N. (1995). Psychological stressors, herpes virus reactivation, and HIV-1 infection. In M. Stein \& A. Baum (Eds.), AIDS and oncology: Perspectives in behavioral medicine (pp. 135-168). Mahwah, NJ: Lawrence Erlbaum Associates, Inc.

Antoni, M. H., Goodkin, K., Goldstein, D., LaPerriere, A., Ironson, G., \& Fletcher, M. A. (1991). Coping responses to HIV-1 serostatus notification predict short-term and long-term affective distress and one-year immunologic status in HIV-1 seronegative and seropositive gay men, Psychosomatic Medicine, 4, 227.

Baker, J. O. (2008). An investigation of the sociological patterns of prayer frequency and content. Sociology of Religion, 69, 169-185.

Bartone, P. T., Ursano, R. J., Wright, K. M., \& Ingraham, L. H. (1989). The impact of a military disaster on the health of assistance workers: A prospective study. The Journal of Nervous and Mental Disease, 177, 317-328. 
Beamon, K. K. (2008). “Used goods": Former African American college student-athletes' perception of exploitation by Division I universities. The Journal of Negro Education, 77, 352-364.

Beatty, D. L., Kamarck, T. W., Matthews, K. A., \& Shiffman, S. (2011). Childhood socioeconomic status is associated with psychosocial resources in African Americans: The Pittsburg Healthy Heart Project. Health Psychology, 30, 472-480.

Bell, N. T., Johnson, S. R., \& Petersen, J. C. (2009). Strength of religious faith of athletes and nonathletes at two NCAA Division III institutions. Sport Journal, 12, 1-10.

Berg, B. L. (2004). A dramaturgical look at interviewing. In B. L. Berg, Qualitative research methods for the social sciences (pp. 75-122). Boston: Pearson Education.

Bergandi, T. A. (1985). Psychological variables relating to the incidence of athletic injury. International Journal of Sport Psychology, 16, 141-149.

Bianco, T., Malo, S., \& Orlick, T. (1999). Sport injury and illness: Elite skiers describe their experiences. Research Quarterly for Exercise and Sport, 70, 157-169.

Borrill, J., Fox, P., \& Roger, D. (2011). Religion, ethnicity, coping style, and selfreported self-harm in a diverse non-clinical UK population. Mental Health, Religion \& Culture, 14, 259-269.

Boudreau, B., \& Poulin, C. (2009). An examination of the validity of the Family Affluence Scale II (FAS II) in a general adolescent population of Canada. Social Indicators Research, 94, 29-42. doi:10.1007/s11205-008-9334-4.

Boyce, W., Torsheim, T., Currie, C., \& Zambon, A. (2006). The Family Affluence Scale as a measure of national wealth: Validation of an adolescent self-report measure. Social Indicators Research, 78, 473-487. doi:10.1007/s11205-005-1607-6

Brady, S., \& Matthews, K. (2002). The influence of socioeconomic status and ethnicity on adolescents' exposure to stressful life events. Journal of Pediatric Psychology, 27, 575-583.

Cagney, K., Browning, C., \& Wallace, D. (2007). The Latino paradox in neighborhood context: the case of asthma and other respiratory conditions. American Journal of Public Health, 97, 919-925. doi:10.2105/AJPH.2005.071472

Carson, F., \& Polman, R. (2008). ACL injury rehabilitation: A psychological case study of a professional rugby union player. Journal of Clinical Sport Psychology, 2, 7190. 
Carson, F., \& Polman, R. (2010). The facilitative nature of avoidance coping within sports injury rehabilitation. Scandinavian Journal of Medicine \& Science in Sports, 20, 235-240.

Carver, C. S., Pozo, C., Harris, S. D., Noriega, V., Scheier, M. F., Robinson, D. S., Ketcham, A.S., Moffat, F.L., \& Clark, K.C. (1993). How coping mediates the effect of optimism on distress: A study of women with early stage breast cancer. Journal of Personality and Social Psychology, 65, 375-390.

Carver, C. S., Scheier, M. F., \& Weintraub, J. K. (1989). Assessing coping strategies: A theoretically based approach. Journal of Personality and Social Psychology, 56, 267-283.

Chen, E., Miller, G. E., Lachman, M. E., Gruenewald, T. L., \& Seeman, T. E. (2012). Protective factors for adults from low-childhood socioeconomic circumstances: The benefits of shift-and-persist for allostatic load. Psychosomatic Medicine, 74, 178-186. doi:10.1097/PSY.0b013e31824206fd.

Chen, E., Strunk, R. C., Tretheway, A., Schreier, H. M., Maharaj, N., \& Miller, G. E. (2011). Resilience in low-socioeconomic-status children with asthma: Adaptations to stress. The Journal of Allergy and Clinical Immunology, 128, 970-976. doi:10.1016/j.jaci.2011.06.040.

Chenube, O. O., \& Omumu, F. C. (2011). The influence of socio-economic status and education in grief experiences and coping strategies employed among widows. Gender \& Behavior, 9, 3612-3623.

Cho, H., \& Khang, Y. (2010). Family Affluence Scale, other socioeconomic position indicators, and self-rated health among South Korean adolescents: Findings from the Korea Youth Risk Behavior Web-based Survey (KYRBWS). Journal of Public Health, 18, 169-178. doi:10.1007/s10389-009-0299-9.

Chibnall, J. T., Tait, R.C., Andresen, E. M., \& Hadler, N. M. (2005). Race and socioeconomic differences in post-settlement outcomes for African American and Caucasian workers' compensation claimants with low back injuries. Pain, 114, 462-472.

Christensen, U., Schmidt, L., Hougaard, C., Kriegbaum, M., \& Holstein, B. (2006). Socioeconomic position and variations in coping strategies in musculoskeletal pain: A cross-sectional study of 128740 - and 50-year old men and women. Journal of Rehabilitation Medicine, 38, 316-321. doi:10.1080/16501970600766467 
Chuang, Y. C., Ennett, S. T., Bauman, K. E., \& Foshee, V. A. (2005). Neighborhood influences on adolescent cigarette and alcohol use: Mediating effects through parent and peer behaviors. Journal of Health \& Social Behavior, 46, 187-204.

Coakley, J. (2009). Sports in society: Issues and controversies (10th ed). New York: McGraw-Hill.

Compas, B. E. (1987). Coping with stress during childhood and adolescence. Psychological Bulletin, 101, 393-403.

Conway, F., Magai, C., McPherson-Salandy, R , \& Milano, K. (2010). Synergy between molecular and contextual views of coping among four ethnic groups of older adults. International Journal of Aging and Human Development, 70, 319-343.

Côté, J., Salmela, J., Baria, A., \& Russell, S. (1993). Organizing and interpreting unstructured qualitative data. Sport Psychologist, 7, 127-137.

Creswell, J. (2007). Philosophical, paradigm, and interpretive Frameworks. In J. C. Creswell, Qualitative Inquiry \& Research Design (2 ${ }^{\text {nd }}$ ed.) (pp. 15-34). Thousand Oaks: Sage.

Currie, C. E., Elton, R. A., Todd, J., \& Platt, S. (1997). Indicators of socioeconomic status for adolescents: The WHO health behaviour in school-aged children survey. Health Education Research, 12, 385-397. doi:10.1093/her/12.3.385.

Currie, C. E., Morgan, A., Rasmussen, V. B., Roberts, C., Samdal, O., \& Settertobulte, W., \& Smith, R. (2004). Young people's health in context. Copenhagen, Denmark: WHO Regional Office for Europe.

Cutts, B., Darby, K., Boone, C., \& Brewis, A. (2009). City structure, obesity, and environmental justice: An integrated analysis of physical and social barriers to walkable streets and park access. Social Sciences \& Medicine, 69, 1314-1322. doi:10.1016/j.socscimed.2009.08.020

Dearnley, C. (2005). A reflection on the use of semi-structured interviews. Nurse Researcher, 13, 19-28.

Dupre, M. E., \& George, L. K. (2011). Exceptions to the rule: Exceptional health among the disadvantaged. Research on Aging, 33, 115-144. doi:10.1177/0164027510391988

Dyck, N. (2006). Athletic scholarships and the politics of child rearing in Canada. Anthropological Notebooks, 12, 65-78. 
Ellis, A. (1994). Reason and emotion in psychotherapy: A comprehensive method of treating human disturbances. Secaucus, NJ: Birch Lane.

Ellison, C. G., Boardman, J. D., Williams, D. R., \& Jackson, J. S. (2001). Religious involvement, stress, and mental health: Findings from the 1995 Detroit Area Study. Social Forces, 80, 215-249.

Ellison, C. G., Musick, M. A., \& Henderson, A. K. (2008). Balm in Gilead: Racism, religious involvement, and psychological distress among African-American adults. Journal for the Scientific Study of Religion, 47, 291-309.

doi:10.1111/j.1468-5906.2008.00408.x

Endler, N. S., \& Parker, J. D. A. (1990). Coping Inventory for Stressful Situations (CISS): Manual. Toronto, Ontario: Multihealth Systems, Inc.

Farmer, M. M., \& Ferraro, K. F. (2005). Are racial disparities in health conditional on socioeconomic status? Social Science \& Medicine, 60, 191-204.

Fiscella, K., Franks, P., Gold, M. R., \& Clancy, C. M. (2008). Inequality in quality: Addressing socioeconomic, racial, and ethnic disparities in health care. Journal of the American Medical Association, 283, 2579- 2584.

Fisher, L. A., Roper, E. A., \& Butryn, T. M. (2009). Engaging cultural studies and traditional sport psychology. In R. J. Schinke \& S. J. Hanrahan (Eds.), Cultural sport psychology (pp. 23-31). Champaign, IL: Human Kinetics.

Ford, I., \& Gordon, S. (1999). Coping with sport injury: Resource loss and the role of social support. Journal of Personal \& Interpersonal Loss, 4, 243-255.

Galli, N., \& Reel, J. J. (2012a). 'It was hard, but it was good': A qualitative exploration of stress-related growth in Division I intercollegiate athletes. Qualitative Research in Sport, Exercise \& Health, 4, 297-319.

Galli, N., \& Reel, J. J. (2012b). Can good come from bad? An examination of adversarial growth in Division I NCAA athletes. Journal of Intercollegiate Sport, 5, 199-212.

Gallup, G. H., \& Lindsay, M. D. (1999). Surveying the religious landscape: Trends in U.S. beliefs. Harrisburg, PA: Morehouse Publishing.

Gary, K., Arango-Lasprilla, J., \& Stevens, L. (2009). Do racial/ethnic differences exist in post-injury outcomes after TBI? A comprehensive review of the literature. Brain Injury, 23(10), 775-789. 
Gould, D., Eklund, R. C., \& Jackson, S. A. (1992). Coping strategies used by U.S. Olympic wrestlers. Research Quarterly for Exercise and Sport, 64, 83-93.

Gould, D., Udry, E., Bridges, D., \& Beck, L. (1997). How to help elite athletes cope psychologically with season-ending injuries. Athletic Therapy Today, 2, 379-399.

Graves, J. L. (2002). The emperor's new clothes: Biological theories of race at the millennium. New Brunswick, NJ: Rutgers University Press.

Green, S. L., \& Weinberg, R. S. (2001). Relationships among athletic identity, coping skills, social support, and psychological impact of injury in recreational participants. Journal of Applied Sport Psychology, 13, 40-59.

Hagger, M. S., Chatzisarantis, N. D., Griffin, M., \& Thatcher, J. (2005). Injury representations, coping, emotions, and functional outcomes in athletes with sports-related injuries: A test of self-regulation theory. Journal of Applied Social Psychology, 35, 2345-2374.

Harrison, C. K., \& Lawrence, S. M. (2004). College students' perceptions, myths, and stereotypes about African American athletes: A qualitative investigation. Sport, Education, and Society, 9, 33-52.

Heijne, A. A., Axelsson, K. K., Werner, S. S., \& Biguet, G. G. (2008). Rehabilitation and recovery after anterior cruciate ligament reconstruction: Patients' experiences. Scandinavian Journal of Medicine \& Science in Sports, 18, 325-335.

Henderson, J. (1999). Suicide in sport: Are athletes at risk? In D. Pargam (Ed.), Psychological bases of sport injuries (2nd Ed.) (pp. 287-302). Morgantown, WV: Fitness Information Technology.

Hoar, S., \& Flint, F. (2008). Determinants of help-seeking intentions in the context of athletic injury recovery. International Journal of Sport \& Exercise Psychology, $6,157-175$.

Holt, N. L., Kingsley, B. C., Tink, L. N., \& Scherer, J. (2011). Benefits and challenges associated with sport participation by children and parents from low-income families. Psychology of Sport and Exercise, 12, 490-499.

Huang, C. (1995). Hardiness and stress: A critical review. Maternal-Child Nursing Journal, 23, 82-89.

Ivey, A. E., D'Andrea, M., Ivey, M. B., \& Simek-Morgan, L. (2001). Theories of counseling and psychotherapy: A multicultural perspective (5th Edition). Boston: Allyn \& Bacon. 
Iwasaki, Y. (2006). Counteracting stress through leisure coping: A prospective health study. Psychology, Health, \& Medicine, 11, 209-220.

James, S. A. (1993). Racial and ethnic differences in infant mortality and low birth weight: A psychosocial critique. Annals of Epidemiology, 3, 130-136.

Jarvis, M. J., \& Wardle, J. (1999). Social patterning of individual health behaviours: The case of cigarette smoking. In M. Marmot, \& R. Wilkinson (Eds.), Social determinants of health (pp. 240-255). Oxford: University Press.

Kahn, R. L., Wolfe, D. M., Quinn, R. P., Snoek, J. D., \& Rosenthal, R. A. (1964). Organizational stress: Studies in role conflict and ambiguity. New York: Wiley.

Kamphoff, C., Gill, D., Araki, K., \& Hammond, C. (2010). A content analysis of cultural diversity in the association for applied sport psychology's conference programs. Journal of Applied Sport Psychology, 22, 231-245. doi:10.1080/10413201003673153

Keyes, C. L. M. (2009). The Black-White paradox in health: Flourishing in the face of social inequality and discrimination. Journal of Personality, 77, 1677-1706. doi:10.1111/j.1467-6494.2009.00597.x

Krohne, H. W. (1993). Vigilance and cognitive avoidance as concepts in coping research. In H. W. Krohne (Ed.), Attention and avoidance ambiguity (pp. 19-50). Seattle, WA: Hogrefe \& Huber.

Klein, A. (1995). Tender machos: Masculine contrasts in the Mexican baseball league. Sociology of Sport Journal, 12, 370-388.

Kowalski, K. C., \& Crocker, P. R. E. (2001). Development and validation of the coping function questionnaire for adolescents in sport. Journal of Sport and Exercise Psychology, 23, 136-155.

Kristenson, M., Eriksen, H. R., Sluiter, J. K., Starke, D., and Ursin, H. (2004). Psychobiological mechanisms of socioeconomic differences in health. Social Science \& Medicine, 58, 1511-1522.

Kunst, A. E., Groenhof, F., \& Andersen, O. (1999). Occupational class and ischemic heart disease mortality in the United States and 11 European countries. Journal of Public Health, 89, 47-53.

Laberge, S., \& Albert, M. (1999). Conceptions in masculinity and of gender transgressions in sport among adolescent boys: Hegemony, contestation, and social class dynamic. Men and Masculinities, 1, 243-267. 
Lawrence, S. M. (2005). African American athletes' experiences of race in sport. International Review for the Sociology of Sport, 40, 99-110.

Lazarus, R. S. (1993). Coping theory and research: Past, present, and future. Psychosomatic Medicine, 55, 234-247.

Lazarus, R. S. (2000). How emotions influence performance in competitive sports. Sport Psychologist, 14, 229-252.

Lazarus, R. S., \& Folkman, S. (1984). Stress, appraisal, and coping. New York: Springer.

Liao, Y., Cooper, R. S., Cao, G., Durazo-Arvizu, R., Kaufman, J. S., Luke, A., \& McGee, D. L. (1998). Mortality patterns among Hispanics: Findings from the NHIS, 19861990. American Journal of Public Health, 88, 227-232.

Lin, Y. (2011). Assessing the use of the Family Affluence Scale as socioeconomic indicators for researching health inequalities in Taiwan adolescents. Social Indicators Research, 102, 463-475. doi:10.1007/s11205-010-9683-7.

Litman, J. A., \& Lunsford, G. D. (2009). Frequency of use and impact of coping strategies assessed by the COPE inventory and their relationships to post-event health and well-being. Journal of Health Psychology, 14, 982-991. doi:10.1177/1359105309341207

Lykeridou, K., Gourounti, K., Sarantaki, A., Loutradis, D., Vaslamatzis, G., \& Deltsidou, A. (2011). Occupational social class, coping responses and infertility-related stress of women undergoing infertility treatment. Journal of Clinical Nursing, 20, 1971-1980. doi:10.1111/j.1365-2702.2011.03696.x

Mann, T., \& Kato, P. M. (1996). Diversity issues in health psychology. In P. M. Kato \& T. Mann, Handbook of diversity issues in health psychology. (pp.3-18). New York: Plenum Press.

Manuel, J. C., Shilt, J. S., Curl, W. W., Smith, J. A., DuRant, R. H., Lester, L., \& Sinal, S. H. (2002). Coping with sports injuries: An examination of the adolescent athlete. Journal Of Adolescent Health, 31, 391-393. doi:10.1016/S1054-139X(02)00400-7

Massad, S., Javier Nieto, F., Palta, M., Smith, M., Clark, R., \& Thabet, A. (2009). Mental health of children in Palestinian kindergartens: Resilience and vulnerability. Child \& Adolescent Mental Health, 14, 89-96. 
Mead, E. E., Gittelsohn, J. J., Roache, C. C., \& Sharma, S. S. (2010). Healthy food intentions and higher socioeconomic status are associated with healthier food choices in an Inuit population. Journal of Human Nutrition \& Dietetics, 23, 8391. doi:10.1111/j.1365-277X.2010.01094.X

Messner, M. A. (1992a). White men misbehaving: Feminism, Afrocentrism, and the promise of a critical standpoint. Journal of Sport \& Social Issues, 16, 136-143.

Messner, M. A. (1992b). Power at play: Sports and the problem of masculinity. Boston: Beacon Press.

Messner, M. A. (2007). Out of play: Critical essay on gender and sport. Albany, NY: State University of New York Press.

McGannon, K. R., \& Johnson, C. R. (2009). Strategies for reflective cultural sport psychology research. In R. J. Schinke \& S. J. Hanrahan (Eds.), Cultural sport psychology (pp. 35-44). Champaign, IL: Human Kinetics.

McIlvane, J. (2007). Disentangling the effects of race and SES on arthritis-related symptoms, coping, and well-being in African American and White women. Aging and Mental Health, 11, 556-569.

Mitchell, I. (2011). Social support and psychological responses in sport-injury rehabilitation. Sport \& Exercise Psychology Review, 7, 30-44.

Mossakowski, K. (2008). Dissecting the influence of race, ethnicity, and socioeconomic status on mental health in young adulthood. Research on Aging, 30, 649-671.

Morrow, S.L. (2005). Quality and trustworthiness in qualitative research in counseling psychology. Journal of Counseling Psychology, 52, 250-260.

doi:10.1037/0022-0167.52.2.250

Miyata, H., \& Kai, I. (2009). Reconsidering evaluation criteria for scientific adequacy in health care research: An integrative framework of quantitative and qualitative criteria. International Journal of Qualitative Research, 8, 64-75.

Nagy Hesse-Biber, S., \& Leavy, P. (2011). An invitation to qualitative research. In N. Hesse-Biber \& P. Leavy, The practice of qualitative research (2nd ed.) (pp. 3-30). Los Angeles: Sage.

National Center for Education. (2010). Status and trends in the education of racial and ethnic groups [Fact Sheet]. Retrieved from http://www.air.org/files/AIRNCESracial_stats_trends1.pdf 
National Collegiate Athletic Association. (2012a). Field hockey injuries: Data from the 2004/05-2008/09 seasons [Fact sheet]. Retrieved from http://www.ncaa.org/wps/wcm/connect/public/ncaa/health+and+safety/sports+inj uries/resources/field+hockey+injuries

National Collegiate Athletic Association. (2012b). Football injuries: Data from the 2004/05-2008/09 seasons [Fact sheet]. Retrieved from http://www.ncaa.org/wps/wcm/connect/public/ncaa/health+and+safety/sports+inj uries/resources/football+injuries

National Collegiate Athletic Association. (2012c). Men's soccer injuries: Data from the 2004/05-2008/09 seasons [Fact sheet]. Retrieved from http://www.ncaa.org/wps/wcm/connect/public/ncaa/health+and+safety/sports+inj uries/resources/soccer+injuries+men

National Collegiate Athletic Association. (2012d). Women's soccer injuries: Data from the 2004/05-2008/09 seasons [Fact sheet]. Retrieved from http://www.ncaa.org/wps/wcm/connect/public/ncaa/health+and+safety/sports+inj uries/resources/soccer+injuries+women

National Collegiate Athletic Association. (2012e). Women's volleyball injuries: Data from the 2004/05-2008/09 seasons [Fact sheet]. Retrieved from http://www.ncaa.org/wps/wcm/connect/public/ncaa/health+and+safety/sports+inj uries/resources/volleyball+injuries+women

Nicholls, A. R., \& Polman, R. (2007). Coping in sport: A systematic review. Journal of Sport Sciences, 25, 11-31.

Nixon, H. L. (1992). A social network analysis of influences on athletes to play with pain and injuries. Journal of Sport and Social Issues, 13, 14-24.

Nixon, H. L. (1996). Explaining pain and injury attitudes and experiences in sport in terms of gender, race, and sports status factors. Journal of Sport \& Social Issues, 20, 33-44.

Noh, S., Beiser, M., Kaspar, V., Hou, F., \& Rummens, J. (1999). Perceived racial discrimination, depression, and coping: A study of Southeast Asian refugees in Canada. Journal of Health \& Social Behavior, 40, 193-207.

O'Connor, J. (2011). Emotional trauma in athletic injury and the relationship among coping skills, injury severity, and post traumatic stress. Dissertation Abstracts International: Section B. Sciences and Engineering, 71(10-B). 
Omi, M., \& Winant, H. (1994). Racial formation in the United States. New York: Routledge.

Ouwehand, C., de Ridder, D. T. D., \& Bensing, J. M. (2009). Who can afford to look to the future? The relationship between socio-economic status and proactive coping. European Journal of Public Health, 19, 412-417.

Pargman, D. (1999). Sport injuries: An overview of psychological perspectives. Psychological bases of sport injuries. In D. Pargman (Ed.), Psychological bases of sport injuries (2nd ed.) (pp. 3-11). Morgantown, WV: Fitness Information Technology.

Patel, K., Eschbach, K., Ray, L., \& Markides, K. (2004). Evaluation of mortality data for older Mexican Americans: Implications for the Hispanic paradox. American Journal of Epidemiology, 159, 707-715.

Pedersen, P. (1986). The grief response and injury: A special challenge for athletes and athletic trainers. Athletic Training, 21, 312-314.

Pitale, M. (2010). Exploring the emotional impact of acculturation on adolescent development: A qualitative investigation of emotions and coping resources in Hispanic adolescent immigrants. Dissertation Abstracts International: Section B: The Sciences and Engineering, 71(4-B).

Plaatjie, M. R., \& Potgieter, J. R. (2011). Coping strategies of soccer players. South African Journal for Research in Sport, Physical Education and Recreation, 33, 107-115.

Podlog, L., \& Dionigi, R. (2010). Coach strategies for addressing psychosocial challenges during the return to sport from injury. Journal of Sports Sciences, 28, 1197-1208. doi:10.1080/02640414.2010.487873

Podlog, L., \& Eklund, R. C. (2006). A longitudinal investigation of competitive athletes' return to sport following serious injury. Journal of Applied Sport Psychology, 18, 44-68. doi:10.1080/10413200500471319

Potter, B. K., Speechley, K. N., Koval, J. J., Gutmanis, I. A., Campbell, K., \& Manuel, D. (2005). Socioeconomic status and non-fatal injuries among Canadian adolescents: Variations across SES and injury measures. BMC Public Health, 5, 132-143. doi:10.1186/1471-2458-5-132

Prenda, K. M., \& Lachman, M. E. (2001). Planning for the future: A life management strategy for increasing control and life satisfaction in adulthood. Psychology and Aging, 67, 898-909. 
Puente-Díaz, R., \& Anshel, M.H. (2005). Sources of acute stress, cognitive appraisal, and coping strategies among highly skilled Mexican and U.S. competitive tennis players. The Journal of Social Psychology, 145, 429-446.

Quinn, A., \& Fallon, B. (1999). The changes in psychological characteristics and reactions of elite athletes from injury onset until full recovery. Journal of Applied Sport Psychology, 11, 210-229.

Quinn, A., \& Fallon, B. (2000). Predictors of recovery time. Journal of Sport Rehabilitation, 9, 62-76.

Ram, N., Starek, J., \& Johnson, J. (2004). Race, ethnicity, and sexual orientation: Still a void in sport and exercise psychology? Journal of Sport \& Exercise Psychology, $26,250-268$.

Rees, T., Mitchell, I., Evans, L., \& Hardy, L. (2010). Stressors, social support and psychological responses to sport injury in high- and low-performance standard participants. Psychology of Sport \& Exercise, 11, 505-512.

Rimmer, J. H. (2005). The conspicuous absence of people with disabilities in public fitness and recreation facilities: Lack of interest or lack of access? American Journal of Health Promotion, 19, 327-329.

Rodgers, W. M. (2008). Understanding the Black and White earnings gap: Why do African Americans continue to earn less despite dramatic gains in education? The American Prospect. Retrieved from http://prospect.org/article/understandingblack-white-earnings-gap

Roth, S., \& Cohen, L. J. (1986). Approach, avoidance, and coping with stress. American Psychologist, 41, 813-819.

Ryba, T. V. (2009). Understanding your role in cultural sport psychology. In R. J. Schinke \& S. J. Hanrahan (Eds.), Cultural sport psychology (pp. 35-44). Champaign, IL: Human Kinetics.

Ryba, T. V., \& Wright, H. K. (2010). Sport psychology and the cultural turn: Noted toward cultural praxis. In T. V. Ryba, R. J. Schinke, \& G. Tenenbaum (Eds.), The cultural turn in sport psychology (pp. 3-27). Morgantown, WV: Fitness Information Technology.

Sanders, M. R., \& Mahalingam, R. (2012). Under the radar: The role of invisible discourse in understanding class-cased privilege. Journal Of Social Issues, 68, 112-127. doi:10.1111/j.1540-4560.2011.01739.x 
Santiago, C. D., \& Wadsworth, M. E. (2009). Coping with family conflict: What's helpful and what's not for low-income adolescents. Journal of Child \& Family Studies, 18, 192-202. doi:10.1007/s10826-008-9219-9

Schoendorf, K. C., Hogue, C. R., Kleinman, J. C., \& Rowley, D. (1992). Mortality among infants of black as compared with white college-educated persons. New England Journal of Medicine, 326, 1522-1526.

Seidman, I. E. (1991). Interviewing as qualitative research: A guide for researchers in education and the social sciences. New York: Teachers College Press.

Selye, H. (1974). Stress without distress. New York: Signet.

Selye, H. (1983). The stress concept: Past, present, and future. In C. L. Cooper (Ed.), Stress research (pp. 1-20). New York: John Wiley \& Sons.

Shaw, R. J., \& Pickett, K. E. (2013). The health benefits of Hispanic communities for non-Hispanic mothers and infants: Another Hispanic paradox. American Journal of Public Health, 103, e1-e6.

Shi, H., Yang, X., Huang, C., Zhou, Z., Zhou, Q., \& Chu, M. (2011). Status and risk factors of unintentional injuries among Chinese undergraduates: A cross-sectional study. BMC Public Health, 11, 1-9.

Shinn, C. A. (2002). Fútbol nation: U.S. Latinos and the goal of homeland. In M. HabellPallán, M. Romero (Eds.), Latino/a Popular Culture (pp. 240-251). New York: New York UP.

Shinnar, R. (2008). Coping with negative social identity: The case of Mexican immigrants. Journal of Social Psychology, 148, 553-576

Silk, M. L., \& Andrews, D. L. (2012). Sport and the neoliberal conjecture: Complicating the consensus. In M. L. Silk \& D. L. Andrews (Eds.), Sport and neoliberalism: Politics, consumption, and culture. Philadelphia, PA: Temple University Press.

Simms, M., Fortuny, K., \& Henderson, E. (2009). Racial and ethnic disparities among low-income families [Fact sheet]. Retrieved from http://www.urban.org/url.cfm?ID=411936

Skinner, E., Edge, K., Altman, J., \& Sherwood, H. (2003). Searching for the structure of coping: A review and critique of category systems for classifying ways of coping. Psychological Bulletin, 129, 216-269. 
Smedley, A., \& Smedley, B. D. (2011). Race in North America: Origin and evolution of a worldview (4th ed). Boulder, CO: Westview Press.

Smith, A. M., Scott, S. G., \& Wiese, D. M. (1990). The psychological effects of sports injuries: Coping. Sports Medicine, 9, 352-369.

Smith, C., Wallston, K., Dwyer, K., \& Dowdy, S. (1997). Beyond good and bad coping: A multidimensional examination of coping with pain in persons with rheumatoid arthritis. Annals of Behavioral Medicine, 19, 11-21.

Stephens, C., Neil, R., \& Smith, P. (2012). The perceived benefits and barriers of sport in spinal cord injured individuals: A qualitative study. Disability \& Rehabilitation, 34, 2061-2070.

Storch, E. A., Roberti, J. W., Bravata, E. A., \& Storch, J. B. (2004). Strength of religious faith: A comparison of intercollegiate athletes and non-athletes. Pastoral Psychology, 52, 485-489.

Taylor, R. J., Chatters, L. M., Jayakody, R., \& Levin, J. (1996). Black and White differences in religious participation: A multisample comparison. Journal for the Scientific Study of Religion, 35, 403-410.

Terry, D. J. (1991). Coping resources and situational appraisals as predictors of coping behavior. Journal of Personality and Individual Differences, 12, 1031-1047.

Thorpe, H. (2010). Psychology of extreme sports. In T. V. Ryba, R. J. Schinke, \& G. Tenenbaum (Eds.), The cultural turn in sport psychology (pp. 363-386). Morgantown, WV: Fitness Information Technology.

Tracey, J. (2003). The emotional response to the injury and rehabilitation process. Journal of Applied Sport Psychology, 15, 279-293. doi:10.1080/104132003902137924

Udry, E. (1997). Coping and social support among injured athletes following surgery. Journal of Sport \& Exercise Psychology, 19, 71-90.

Udry, E., Gould, D., Bridges, D., \& Beck, L. (1997). Down but not out: Athlete responses to season-ending injuries. Journal of Sport and Exercise Psychology, 19, 229-248.

Vishnevsky, T., Cann, A., Calhoun, L., Tedeschi, R., \& Demakis, G. (2010). Gender differences in self-reported posttraumatic growth: A meta-analysis. Psychology of Women Quarterly, 34, 110-120. doi:10.1111/j.1471-6402.2009.01546.x 
Wadey, R., Evans, L., Hanton, S., \& Neil, R. (2012). An examination of hardiness throughout the sport-injury process: A qualitative follow-up study. British Journal of Health Psychology, 17, 872-893.

Wenner, L. (1994). The dream team, communicative dirt, and the marketing of synergy: USA basketball and cross-merchandising in television commercials. Journal of Sport and Social Issues, 18, 27-47.

Wiese-Bjornstal, D. M., Smith, A. M., \& LaMott, E. E. (1995). A model of psychologic response to athletic injury and rehabilitation. Athletic Training: Sports Health Care Perspectives, 1, 17-30.

Wiese-Bjornstal, D. M., Smith, A. M., Shaffer, S. M., \& Morrey, M. A. (1998). An integrated model of response to sport injury: Psychological and sociological dynamics. Journal of Applied Sport Psychology, 10, 46-69.

Williams, D. M., \& Lawler, K. A. (2003). Importance of macro social structures and personality hardiness to the stress-related relationship in low-income women. Journal of Human Behavior in the Social Environment, 7, 121-140.

Williams, D. R., \& Collins, C. (1995). US socioeconomic and racial differences in health: Patterns and explanations. Annual Review of Sociology, 21, 349-386.

Williams, D. R., \& Harris-Reid, M. (1999). Race and mental health: Emerging patterns and promising approaches. In A. V. Horwitz \& T. L. Scheid (Eds.), A handbook for the study of mental health: Social context, theories, and systems (pp. 295-314). New York: Cambridge University Press.

Williams, D. R., \& Rucker, T. (1996). Socioeconomic status and the health of racial minority populations. In P. M. Kato \& T. Mann, Handbook of diversity issues in health psychology (pp. 407-423). New York: Plenum Press.

Williams, D. R., Spencer, M. S., \& Jackson, J. S. (1999). Race, stress, and physical health: The role of group identity. In R. J. Contrada, R. D. Ashmore (Eds.), Self, social identity, and physical health: Interdisciplinary explorations (pp. 71-100). New York, NY US: Oxford University Press.

Williams, J. M., \& Andersen, M. B. (1998). Psychosocial antecedents of sport injury: Review and critique of the stress and injury model. Journal of Applied Sport Psychology, 10, 5-25. 
Wold, B., \& Hendry, L. (1998). Social and environmental factors associated with physical activity in young people. In S. Biddle, J. Sallis, N. Cavil (Eds.), Young and active? Young people and health-enhancing physical activity evidence and implications. London: Health Education Authority.

Wrosch, C., Heckhausen, J., Lachman, M.E. (2000). Primary and secondary control strategies for managing health and financial stress across adulthood. Psychology and Aging, 15, 387-399. 


\section{Appendix A}

\section{DEMOGRAPHIC INFORMATION}

Please write the answers to the following questions.

Age:

Gender:

Race/Ethnicity:

Sport:

Amount of athletic scholarship money (if applicable):

Eligibility level in sport:

Date of injury:

Type of injury:

Estimated (or actual) recovery time (from date of injury to expected date of full recovery):

What is the best way to contact you? (phone, email, etc.)

Contact information: 


\section{Appendix B}

FAMILY AFFLUENCE SCALE II

\begin{tabular}{|c|c|c|c|c|}
\hline \multirow[t]{2}{*}{ Item } & \multicolumn{4}{|c|}{ Points per response option } \\
\hline & 0 & 1 & 2 & 3 \\
\hline 1. How many vehicles does your family own? & None & One & \multicolumn{2}{|c|}{ Two or more } \\
\hline $\begin{array}{l}\text { 2. Growing up, did you have your own } \\
\text { bedroom? }\end{array}$ & No & Yes & & \\
\hline $\begin{array}{l}\text { 3. During the past } 12 \text { months, how many times } \\
\text { did you travel away on vacation with your } \\
\text { family? }\end{array}$ & None & Once & Twice & More than twice \\
\hline $\begin{array}{l}\text { 4. How many computers does your family } \\
\text { own? }\end{array}$ & None & One & Two & More than two \\
\hline
\end{tabular}




\section{Appendix C}

\section{INFORMED CONSENT FORM}

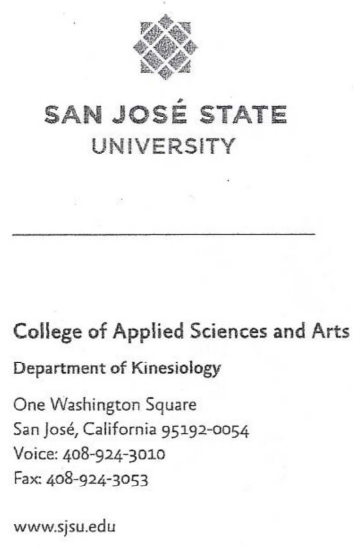

Responsible Investigator: Matthew Bejar (Kinesiology Graduate Student) Title of Study: Influence of socioeconomic status on coping with athletic injury

Dear Student-Athlete,

You have been asked to participate in a pilot research study, which will investigate the influence of socioeconomic status on how college athletes cope with injuries. You will be asked to complete a brief questionnaire, which should take approximately 10 to 15 minutes. Afterwards, you may also be asked to participate in an interview, which may last between 45 and 90 minutes. These interviews will be recorded and transcribed verbatim. This study could potentially encourage further research in sport psychology, which could conceivably improve the approaches coaches, athletic trainers, sport psychologists, and other athletic personnel use to help athletes manage their injuries. There are no serious risks that are foreseen with your participation in this study.

The results of this study will be presented in a Masters' thesis. If you participate in an interview, you will be assigned a pseudonym in the manuscript in order to protect your identity. While these results will be presented in the final paper, no identifying information will be included.

If you have any questions about this study, you may contact Matthew Bejar at matthew.bejar@sjsu.edu. Complaints of this research may be presented to Shirley Reekie, Ph.D., Chair of the Kinesiology Department, at (408) 924-3010 or shirley.reekie@sjsu.edu. Questions about a research subject's rights or research-related injury may be presented to Pamelä Stacks, Ph.D., Associate Vice President, Graduate Studies and Research, at (408) 924-2427.

Your participation in this study is voluntary. You may refuse to participate in the entire study or in any part of the study. You have the right to not answer questions you do not wish to answer. If you decide to participate in the study, you are free to withdraw at any time without any negative effect on your relations with San José State University.

By signing this consent form, you acknowledge that you have read this form and have given written consent to participate in this study.

Signature of Participant

Date

Signature of Researcher
Date 


\section{Appendix D}

\section{INTERVIEW GUIDE}

1. Tell me how you got into your sport.

Probe: When did it become most meaningful?

2. Growing up, what opportunities did you have for training?

Probe: Can you discuss any experiences with private club teams or coaching?

Probe: Was there anything you did not have that some of your other teammates had?

3. Tell me about your family life/family background.

Probe: What were some examples of daily hassles when you were growing up? (What stressed you out on a consistent basis?)

Probe: Was there any major hardship (e.g., illness) in the family?

Probe: Were there any other major life events when you were growing up?

How did you deal with it?

Probe: What values do you carry with you from your family or cultural background?

Probe: How meaningful is your family background/culture in everyday life? How meaningful is it in your sport?

Probe: What comes to mind when you think of socioeconomic status or social class?

4. Tell me about your recent injury.

Probe: What was your initial reaction to being injured?

Probe: How did you deal with it?

Probe: How did this change over time?

Probe: Given that the study is about socioeconomic status, how did that weigh in?

5. Describe the kind of feedback and/or support you received from the significant people in your life.

Probe: What kind of support have you received from them?

6. Did you have any concerns that you would lose your scholarship? Discuss. 
7. What aspects of your background influenced the way you dealt with this injury? Probe: What are some values which we discussed earlier that may have had an impact on how you dealt with your injury?

Probe: How has your class and/or family background influenced the way you have managed your injury?

8. Is there anything important to your injury or rehabilitation that we have not discussed? 
Appendix E

PARTICIPANT PROFILES

\begin{tabular}{ccccccc}
\hline Pseudonym & Age & Gender & Race/Ethnicity & Year & Sport & SCH \\
\hline Sam & 19 & Male & African American & Freshman & Football & $100 \%$ \\
Russell & 19 & Male & African American & Sophomore & Football & $100 \%$ \\
Brett & 21 & Male & African American & Junior & Football & $100 \%$ \\
Richard & 20 & Male & Pacific Islander & Junior & Football & $100 \%$ \\
Catherine & 19 & Female & Filipino/Japanese & Sophomore & Gymnastics & $100 \%$ \\
Andy & 21 & Male & White & Junior & Football & $100 \%$ \\
Simon & 21 & Male & African American & Junior & Football & $100 \%$ \\
Ian & 21 & Male & African American & Junior & Football & $100 \%$ \\
Michael & 19 & Male & African American & Freshman & Football & $100 \%$ \\
Emily & 22 & Female & White & Sophomore & Tennis & $100 \%$ \\
Tony & 18 & Male & Hispanic & Freshman & Soccer & $35 \%$ \\
\hline
\end{tabular}

Note. $\mathrm{SCH}=$ percentage of tuition and other academic-related costs covered by participant's athletic scholarship. 


\section{PARTICIPANT PROFILES (CONTINUED)}

\begin{tabular}{cccc}
\hline Pseudonym & Injury & Recovery Time (weeks) & FAS II \\
\hline Sam & torn meniscus & 13 & 3 \\
Russell & fractured clavicle & 9 & 5 \\
Brett & torn TFCC (wrist) & 39 & 5 \\
Richard & torn ACL & 35 & 4 \\
Catherine & ruptured Achilles & 26 & 3 \\
Andy & FAI (hip) & 17 & 5 \\
Simon & torn UCL & 13 & 5 \\
Ian & torn labrum & 22 & 3 \\
Michael & torn labrum & 22 & 5 \\
Emily & strained hamstring & 4 & 4 \\
Tony & broken foot & 8 & 5 \\
\hline Note. FAS II = participant's score on the Family Affluence Scale II (Currie et al., \\
2004), TFCC $=$ triangular fibrocartilage complex, ACL= anterior cruciate ligament, \\
FAI = femoral acetabular impingement, UCL = ulnar collateral ligament.
\end{tabular}


Appendix F

CONTENT ANALYSIS RESULTS

Raw Data Themes

-confused

-shocked

-didn't know what happened

-didn't think it was serious

-never going to play again

-will never be the same

-why does this always

happen

-won't be a starter anymore

-going to miss season

-nothing positive in life

-sad

-depressed

-bummed

-got down

-felt like crying

$-\operatorname{mad}$

-discouraged

-felt weak

-couldn't perform simple

tasks

-felt like forever

-knew something was

wrong

-needed some time off

-glad surgery fixed problem
Higher Order Themes General Dimensions
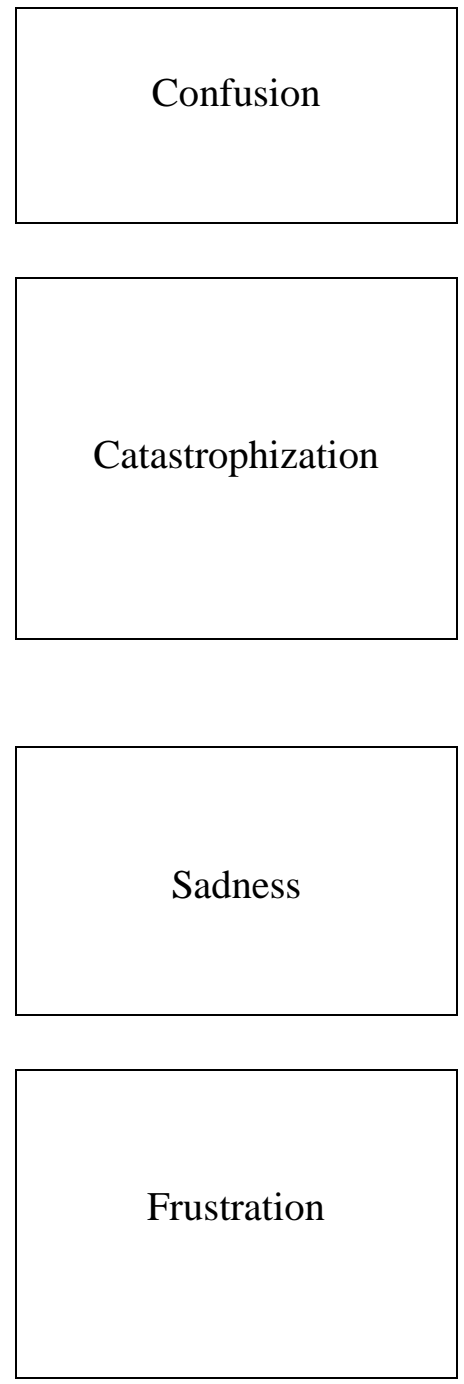

Relief
Cognitive Appraisals

Emotional Reactions
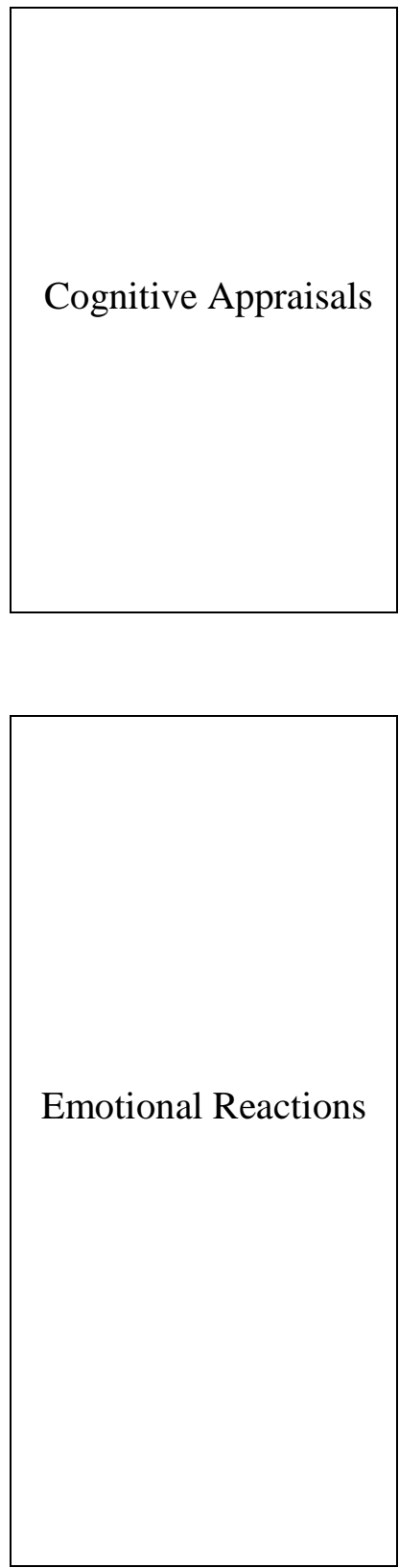


\section{Raw Data Themes}

-been through this before

-been hurt before

-learned from past

experiences

-been through worse things

-did whatever trainer said -did everything to play again -did research on injury -didn't want to sit around

-couldn't give up

-stayed tough

-kept head up

-fought through it

-pushed when times were

hard

-didn't want to end up back

on streets

-had to be strong for family

-family/friends prepared meals

-family/friends helped with getting dressed -driven to doctor's appointments -parents paid medical bills -coaches assisted with rehabilitation exercises

-advice from teammates with same injury -parents did research on injury
Higher Order Themes

General Dimensions
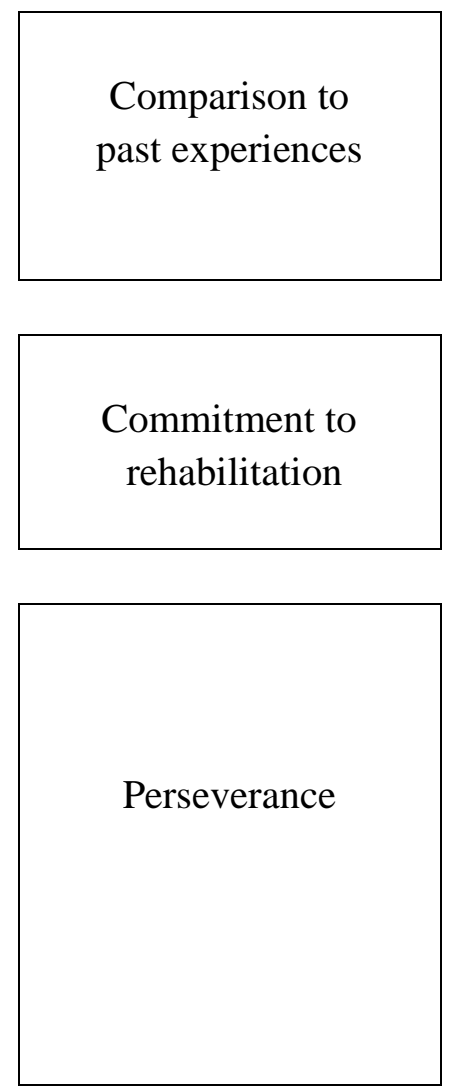

Problem-focused

Coping

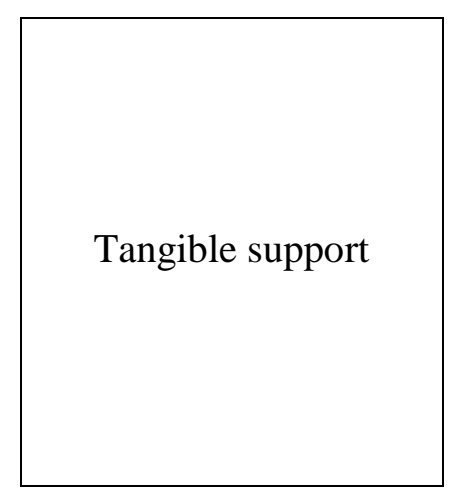

Informational support 
Raw Data Themes

-viewed injury as a

learning experience

-saw more good than

negative

-able to develop other

areas of body

-able to spend more time

learning strategy

-optimistic attitude/

thoughts

-grateful that school and

medical bills are paid for

-prayed

-became closer to God

-had faith in God

-family visited

-reassurance from family/

friends

-family offered prayers

-motivation from family

and teammates

-family was warm and

caring

-coach assured scholarship

was safe

-coach expressed

confidence in recovery

-laughed about injury with

other injured teammates
Higher Order Themes

General Dimensions
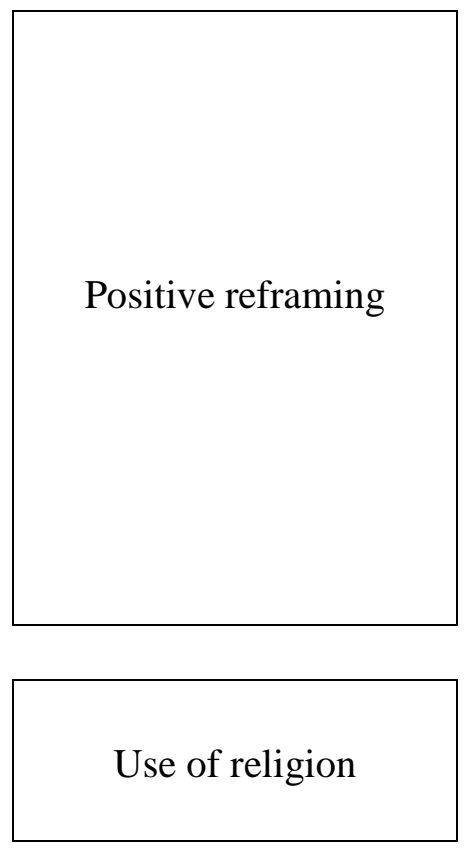

Emotion-focused

Coping

Emotional support

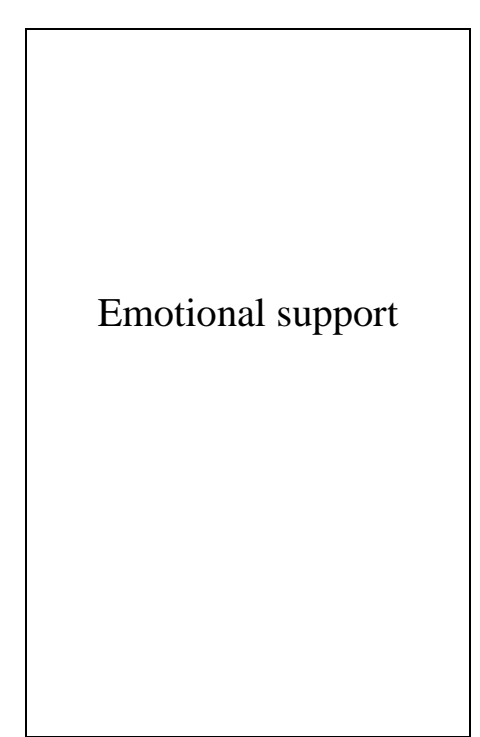


Raw Data Themes

\begin{tabular}{l}
\hline -ignored pain \\
-played through injury \\
-finished game/match \\
-pressure from coach and \\
teammates to play
\end{tabular}

\begin{tabular}{|l|}
\hline -avoided teammates \\
-did not want parent(s) to \\
worry \\
-shut self off from others \\
-just wanted to sleep \\
\hline
\end{tabular}

-needed to stay occupied -participated in other activities

-family/significant other helped keep mind off of injury
Higher Order Themes
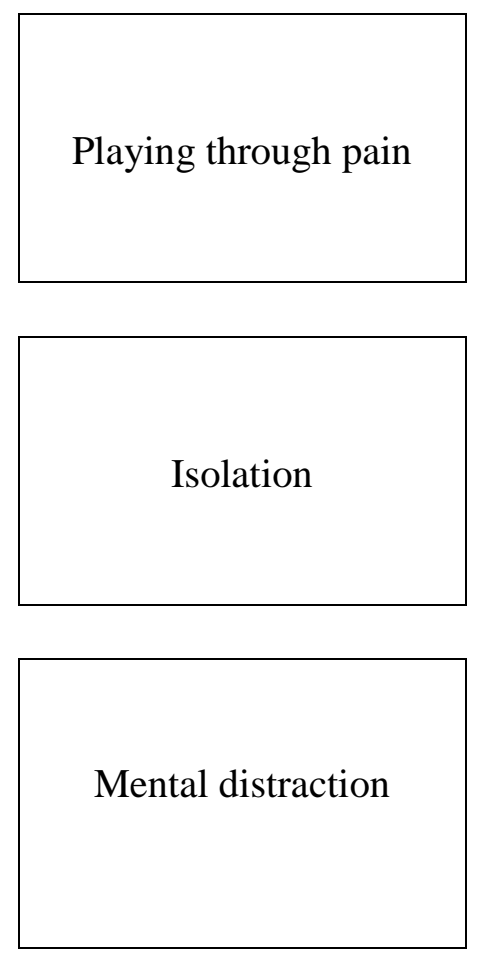

General Dimensions

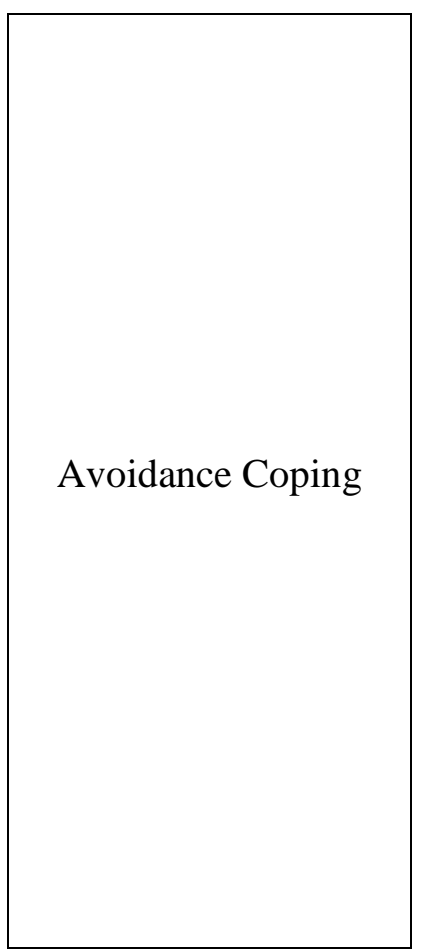




\section{Appendix G}

\section{HUMAN SUBJECTS-INSTITUTIONAL REVIEW BOARD APPROVAL}

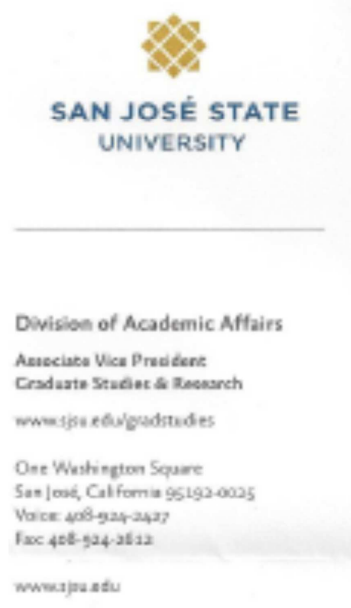

To: Matthew Bejar

From: Pamela Stacks, Ph.D.

Associate Vice President

Graduate Studies and Research

Date: January 23, 2013

The Human Subjects-Institutional Review Board has approved your request to use human subjects in the study entitled:

"Influence of socioeconomic status on coping with athletic injury"

This approval is contingent upon the subjects participating in your research project being appropriately protected from risk. This includes the protection of the confidentiality of the subjects' identity when they participate in your research project, and with regard to all data that may be collected from the subjects. The approval includes continued monitoring of your research by the Board to assure that the subjects are being adequately and properly protected from such risks. If at any time a subject becomes injured or complains of injury, you must notify Dr. Pamela Stacks, Ph.D. immediately. Injury includes but is not limited to bodily harm, psychological trauma, and release of potentially damaging personal information. This approval for the human subject's portion of your project is in effect for one year, and data collection beyond January 23,2014 requires an extension request.

Please also be advised that all subjects need to be fully informed and aware that their participation in your research project is voluntary, and that he or she may withdraw from the project at any time. Further, a subject's participation, refusal to perticipate, or withdrawal will not affect any services that the subject is receiving or will receive at the institution in which the research is being conducted.

If you have any questions, plense contact me at (408) 924-2427.

Protocol \# S1302010

ce. Ted Butryn

0054 